\title{
Hydrogen diffusivity and solubility in stressed fcc crystals.
}

\author{
Damien Connétable ${ }^{1, *}$ and Philippe Maugis $^{2, \dagger}$ \\ ${ }^{1}$ CIRIMAT, UMR 5085, CNRS INP UPS, ENSIACET 4, \\ allée Émile Monso, BP 44362, F-31030 Toulouse Cedex 4, France \\ ${ }^{2}$ Aix Marseille Univ, CNRS, IM2NP, Marseille, France
}

(Dated: May 11, 2021)

\begin{abstract}
Although many materials are used under extreme conditions, the effects of stress and strain are often neglected in material studies, especially when studying the solubility or calculating the diffusivity of substitutional or interstitial species. In this work, a general method based on firstprinciples calculations and elasticity theory in fcc systems is presented to fill this gap. The case of hydrogen in aluminum is investigated in detail as an application sample by comparing results from the density functional theory (DFT) and the elasticity theory. Additional systems, $\mathrm{Ni}, \mathrm{Cu}$ and $\mathrm{Pd}$, are also examined for hydrogen but in the framework of the elasticity theory only. Different types of stresses, i.e. hydrostatic, multi-axial and shear stress, are investigated for comparison purposes. The symmetry break induced by the loading is analyzed at the atomic scale by calculating the jump rates, at macroscopic scale from computed diffusion coefficients. Equations of diffusion are developed for each loading. Results show that the effect of loading on atomic parameters -insertion energies, energy barriers, etc.- can be accurately captured by the elasticity theory in terms of elementary parameters calculated using the DFT. Results show that the effect of stress is weak in the case of hydrogen.
\end{abstract}

\section{INTRODUCTION}

Understanding the thermodynamic and kinetic processes associated with the different species in a solid solution is at the heart of many material science problems, as they address issues related to the prediction of time evolution and ageing of structural materials. In this work, hydrogen embrittlement (HE) is at the heart of the question. Metals suffer from the deleterious effects of hydrogen, this limits their uses in many potential applications. There are plenty examples associated with the $\mathrm{HE}$ in literature [1, 2]. Hydrogen embrittlement of metallic systems is a rich and complex field of research. Many mechanisms have been proposed to explain HE: models such as hydrogen-enhanced localizedplasticity (HELP) [3-5], decohesion (HEDE) [6, 7] or the adsorption-induced dislocation emission (AIDE) [8]. In these models, the main factors responsible for the intragranular embrittlement of metallic systems are vacancies $[9,10]$, dislocations [11], and structural defects [12]. Various theoretical works carried out in recent years on the interaction of hydrogen with vacancies confirmed these hypotheses and showed, for example, that depending on experimental conditions, hydrogen-vacancy clusters can form in large quantities, thus weakening the metal. For instance, in the case of face-centered cubic metal systems (i.e. $\mathrm{Al}, \mathrm{Ni}, \mathrm{Pd}$ ), two behaviors were observed: either i) these vacancy-hydrogen clusters have a low impact on $\mathrm{HE}$, as in nickel, (this would require very high hydrogen concentrations at intermediate temperatures, i.e. 300$600 \mathrm{~K}$, for the effect to be observable) [13-16], or ii) these clusters have a high impact, as in the $\mathrm{Al}$ system (a very

\footnotetext{
* damien.connetable@ensiacet.fr

$\dagger$ philippe.maugis@im2np.fr
}

large amount of clusters even at low $\mathrm{H}$ concentrations) $[13,17]$. In the case of grain boundaries $[14,18,19]$ or dislocations [20] equivalent works were also carried out to understand and investigate the interactions between hydrogen and extended defects.

In these studies however, DFT calculations were almost always performed at zero pressure (stress-free), with the aim of predicting hydrogen solubility and diffusivity under normal pressure conditions. In the specific case of fcc systems, i.e. copper [13, 21], palladium [13, 22], aluminum [17, 23] and nickel [16], numerous studies were carried out to evaluate the solubility as well as the diffusivity of interstitials and particularly hydrogen. In these studies, insertion sites as well as diffusion mechanisms were studied. There are even more sophisticated studies where tunneling effects at low temperatures were taken into account $[24,25]$. However, real metal systems are subject to external and internal mechanical stresses (due to temperature, precipitates, grain boundaries, etc.) which can be significant during fabrication steps and/or service life.

The objective of this paper is twofold: on the one hand to propose a simple way to evaluate the effect of stresses on the solubility of $\mathrm{H}$, and on the other hand to easily calculate the effect of stresses on the diffusion coefficient. This study is intended to be generalizable and directly transposed to other interstitial species (carbon, oxygen or nitrogen, for example) insofar as the processes at the atomic scale are identical to those presented here, otherwise adjustments will have to be made in particular to calculate the diffusion coefficient. Recently, several studies have been carried out to investigate the effect of stresses [26]. However, these studies focused on either investigating deformations by means of DFT calculations without correlating these results to the theory of elasticity [27, 28], or conversely [29], or using this latter theory 
to study the solubility and diffusivity of insertion elements [17, 30]. Regarding bcc systems, Maugis [31-34] published many studies using this coupling to investigate the effect of stresses on phase transitions. Moreover, the benefits of using the theory of elasticity to study defects, or interstitials in the presence of extended defects, are already documented in the literature [26, 35-38]. In the case of fcc systems, there is no study using such an approach except a recent work on vacancies [39].

In this work, the DFT approach, which implies precise but heavy numerical calculations, will be compared to the theory of elasticity in continuous media. This latter approach has the advantage of being able to characterize the effect of a stress on the concentration and diffusion coefficient of $\mathrm{H}$ in metals while requiring few quantities calculated using DFT simulations. The theoretical case of $\mathrm{Al}$ will help highlight the strength of this simplification. Different types of loadings were studied: along one, two or three main directions, hydrostatic stress, shear stress and along the [111] direction. Diffusion equations are given for all these cases. The case of the $\mathrm{H}$ diffusivity was considered, taking into account only direct jumps between octahedral sites and first neighboring tetrahedral sites. Indeed, in previous studies [16], it was clearly shown that direct jumps between two octahedral sites or between two tetrahedral sites lead to a single transition state, which is then a so-called second-order transition state which only has a weak impact on the diffusion coefficient, even at high temperatures.

In order to do this, the article is organized as follows. Section II is dedicated to the method. More particularly, to how an applied stress leads to a lifting of degeneracy on the diffusion coefficients. The elasticity approach is then described in Section III. The DFT procedure is presented in Section IV. Section V gathers parameters used in the elasticity theory and ground-state properties of fcc systems. Lastly, the effects of stress on solubility and diffusivity in fcc metals are discussed in Section VI.

\section{METHODOLOGY}

This section summarizes the procedure for taking account of the effect of symmetry break, due to loading in fcc systems, on the solubility and diffusivity of an interstitial atom. The following results can be applied to any interstitial species (i.e. $\mathrm{H}, \mathrm{C}, \mathrm{N}$ and $\mathrm{O}$ ) that is stable in octahedral and tetrahedral sites. The macroscopic diffusion equations, as well as the atomistic mechanisms, are therefore presented in different cases. From these results, new interstitial diffusion equations in fcc distorted structures with symmetry break are discussed. Five loading scenarios were considered: hydrostatic loading, uni-axial loadings -in the [001] crystallographic direction and in the [111] direction-, bi-axial and tri-axial loadings, and shear loading in plane (001).

\section{A. Hydrostatic loading}

Under hydrostatic loading, the symmetry of the X-fcc system is preserved, i.e. the structure still belongs to space group $225, F m \overline{3} m$, where X metal atoms occupy $4 a$ positions. Studies $[13,16,23]$ show that, in fcc systems, $\mathrm{H}$ atoms preferably occupy either octahedral (labeled $o$ ) or tetrahedral (labeled $t$ ) sites. For each $\mathrm{X}$ atom, there is one $o$ site, located in a $4 b$ sites, and two $t$ sites in $8 c$. Atomic positions and the geometry of the system are summarized in Table I.

To calculate the concentration of hydrogen in the solid solution, $C_{H}(p, T)$, Sieverts' law [41] can be used. $C_{H}(p, T)$ depends on the external loading, here the pressure $p$, and the temperature, $T$. It is also a function of the insertion energies into the two sites, $t$ and $o$. It is therefore given by:

$$
\begin{aligned}
& C_{H}(p, T)=\sqrt{\frac{p_{H_{2}}}{p_{o}}} {\left[2 \exp \left[-\frac{G_{\text {ins }}^{\mathrm{t}}(p, T)}{k_{B} T}\right]\right.} \\
&\left.+\exp \left[-\frac{G_{\mathrm{ins}}^{\mathrm{o}}(p, T)}{k_{B} T}\right]\right]
\end{aligned}
$$

where $G_{\text {ins }}^{s}(p, T)$ is the Gibbs energy of hydrogen insertion in the $s$ site, i.e. in the $t$ and $o$ sites (see below). The aim of this work is to rationalize each contribution in order to calculate $G_{\text {ins }}^{s}(p, T)$ (see Sections III and IV). Hydrogen atoms are here assumed to be dissolved in the metal, in infinite limit dilution, in the insertion sites $(t$ and $o$ ) only. Clustering or segregation at dislocations and grains boundaries were thus neglected. The expression also depends on the external pressure of the gas, $p_{H_{2}}$ ( $p_{o}$ is the pressure reference state equal to $\left.1 \mathrm{~atm}\right) . p_{H_{2}}$ can be different from the stress $p$ induced by the loading.

As found and described in earlier works [16, 17, 42], the diffusion coefficient, $D(p, T)$, of the interstitial species in fcc structures, valid when $o$ and $t$ sites are both stable, is given by:

$$
D(p, T)=\frac{a^{2}(p, T)}{2} \frac{\Gamma_{o t}(p, T) \Gamma_{t o}(p, T)}{\Gamma_{t o}(p, T)+2 \Gamma_{o t}(p, T)}
$$

To obtain this expression, the Landman's approach [43] was used to obtain the diffusion coefficients. All details are given in the work of David et al. [17]. $a$ is the lattice parameter of the cubic cell, which depends on $p$ and $T$. $\Gamma_{o t}$ (resp. $\Gamma_{t o}$ ) corresponds to the jump rate of an $\mathrm{H}$ atom leaving the $o$ site and jumping directly into the first-nearest $t$ site (resp. from $t$ to $o$ ), as displayed in Fig. 1. The jump rates, $\Gamma_{x y}$, are also a function of pressure and temperature. Their expressions are [44]:

$$
\Gamma_{x y}(p, T)=\frac{k_{B} T}{h} \frac{\mathcal{Z}_{t s}(p, T)}{\mathcal{Z}_{x}(p, T)} \exp \left[-\frac{H_{m}^{x y}(p, T)}{k_{B} T}\right]
$$

where $H_{m}^{x y}$ is the migration enthalpy from $x$ to $y$ positions, $\mathcal{Z}_{t s}$ the (vibration) partition function for the transition state and $\mathcal{Z}_{x}$ the partition function for the initial 
Table I. Wyckoff positions after loading [40]. $a, b$ and $c$ are the lengths of the primitive unit-cells. For convenience purposes, the super-cells used for DFT simulations are based on the tetragonal representation of the cell, see Fig. 1.

\begin{tabular}{|c|c|c|c|c|c|}
\hline $\begin{array}{c}\text { sg } \\
\text { loading }\end{array}$ & $\begin{array}{c}225 \\
\text { hydrostatic }\end{array}$ & $\begin{array}{c}139 \\
\text { uni-axial }[001] / \\
\text { bi-axial }[100]+[010]\end{array}$ & $\begin{array}{c}\text { 166-h } \\
\text { uni-axial along [111] }\end{array}$ & $\begin{array}{c}\quad 69 \\
\text { orthorhombic } \\
\text { tri-axial } \\
\end{array}$ & $\begin{array}{c}15 \\
\text { shear }\end{array}$ \\
\hline unit-cell & $\left.\begin{array}{lll}a & 0 & 0 \\
0 & a & 0 \\
0 & 0 & a\end{array}\right]$ & {$\left[\begin{array}{ccc}-\frac{a \sqrt{2}}{2} & \frac{a \sqrt{2}}{2} & c / 2 \\
\frac{a \sqrt{2}}{2} & -\frac{a \sqrt{2}}{2} & c / 2 \\
\frac{a \sqrt{2}}{2} & \frac{a \sqrt{2}}{2} & -c / 2\end{array}\right]$} & {$\left[\begin{array}{ccc}\frac{a \sqrt{2}}{2} & 0 & 0 \\
-\frac{a \sqrt{2}}{4} & \frac{a \sqrt{6}}{4} & 0 \\
0 & 0 & c\end{array}\right]$} & $\left.\begin{array}{lll}a & 0 & 0 \\
0 & b & 0 \\
0 & 0 & c\end{array}\right]$ & {$\left[\begin{array}{ccc}a & 0 & 0 \\
a \cos (\gamma) & a \sin (\gamma) & 0 \\
0 & 0 & c\end{array}\right]$} \\
\hline $\mathrm{Al}$ & $\begin{array}{l}4 a(0,0,0), \\
(1 / 2,1 / 2,0), \\
(1 / 2,0,1 / 2), \\
(0,1 / 2,1 / 2)\end{array}$ & $\begin{array}{c}2 a(0,0,0), \\
(1 / 2,1 / 2,1 / 2)\end{array}$ & $\begin{array}{c}3 a(0,0,0), \\
(2 / 3,1 / 3,1 / 3), \\
(1 / 3,2 / 3,2 / 3)\end{array}$ & $\begin{array}{l}4 a(0,0,0), \\
(1 / 2,1 / 2,0), \\
(1 / 2,0,1 / 2), \\
(0,1 / 2,1 / 2)\end{array}$ & $\begin{array}{c}4 d(1 / 4,1 / 4,1 / 2) \\
(3 / 4,1 / 4,0) \\
(3 / 4,3 / 4,1 / 2) \\
(1 / 4,3 / 4,0)\end{array}$ \\
\hline$O$ sites & \begin{tabular}{|c|}
$4 b(1 / 2,1 / 2,1 / 2)$, \\
$(0,1 / 2,0)$ \\
$(1 / 2,0,0)$ \\
$(0,0,1 / 2)$ \\
\end{tabular} & $\begin{array}{c}2 b(0,0,1 / 2) \\
(1 / 2,1 / 2,0)\end{array}$ & $\begin{array}{c}3 b(0,0,1 / 2), \\
(2 / 3,1 / 3,5 / 6), \\
(1 / 3,2 / 3,1 / 6)\end{array}$ & $\begin{array}{c}4 b(1 / 2,1 / 2,1 / 2) \\
(0,1 / 2,0) \\
(1 / 2,0,0) \\
(0,0,1 / 2)\end{array}$ & $\begin{array}{c}4 c(1 / 4,1 / 4,0), \\
(3 / 4,1 / 4,1 / 2), \\
(3 / 4,3 / 4,0), \\
(1 / 4,3 / 4,1 / 2)\end{array}$ \\
\hline$t$ sites & \begin{tabular}{|c|}
$8 c(1 / 4,1 / 4,1 / 4)$, \\
$(3 / 4,3 / 4,3 / 4)$, \\
$(3 / 4,1 / 4,1 / 4)$, \\
$(1 / 4,3 / 4,1 / 4)$, \\
$(1 / 4,1 / 4,3 / 4)$, \\
$(3 / 4,3 / 4,1 / 4)$, \\
$(3 / 4,1 / 4,3 / 4)$, \\
$(1 / 4,3 / 4,3 / 4)$ \\
\end{tabular} & $\begin{array}{c}4 d(0,1 / 2,1 / 4), \\
(1 / 2,0,1 / 4), \\
(1 / 2,0,3 / 4), \\
(0,1 / 2,3 / 4)\end{array}$ & $\begin{array}{c}6 c\left(0,0, z_{c}\right) \\
\left(0,0,-z_{h}\right) \\
\left(2 / 3,1 / 3,1 / 3+z_{h}\right), \\
\left(2 / 3,1 / 3,1 / 3-z_{h}\right), \\
\left(1 / 3,2 / 3,2 / 3+z_{h}\right), \\
\left(1 / 3,2 / 3,2 / 3-z_{h}\right)\end{array}$ & $\begin{array}{c}8 f(1 / 4,1 / 4,1 / 4), \\
(3 / 4,3 / 4,3 / 4), \\
(3 / 4,1 / 4,1 / 4), \\
(1 / 4,3 / 4,1 / 4) \\
(1 / 4,1 / 4,3 / 4), \\
(3 / 4,3 / 4,1 / 4) \\
(3 / 4,1 / 4,3 / 4) \\
(1 / 4,3 / 4,3 / 4) \\
\end{array}$ & $\begin{array}{c}4 e\left(0, y_{e}, 3 / 4\right), \\
\left(0,-y_{e}, 3 / 4\right) \\
\left(1 / 2, y_{e}+1 / 2,3 / 4\right), \\
\left(1 / 2,1 / 2-y_{e}, 3 / 4\right)\end{array}$ \\
\hline
\end{tabular}

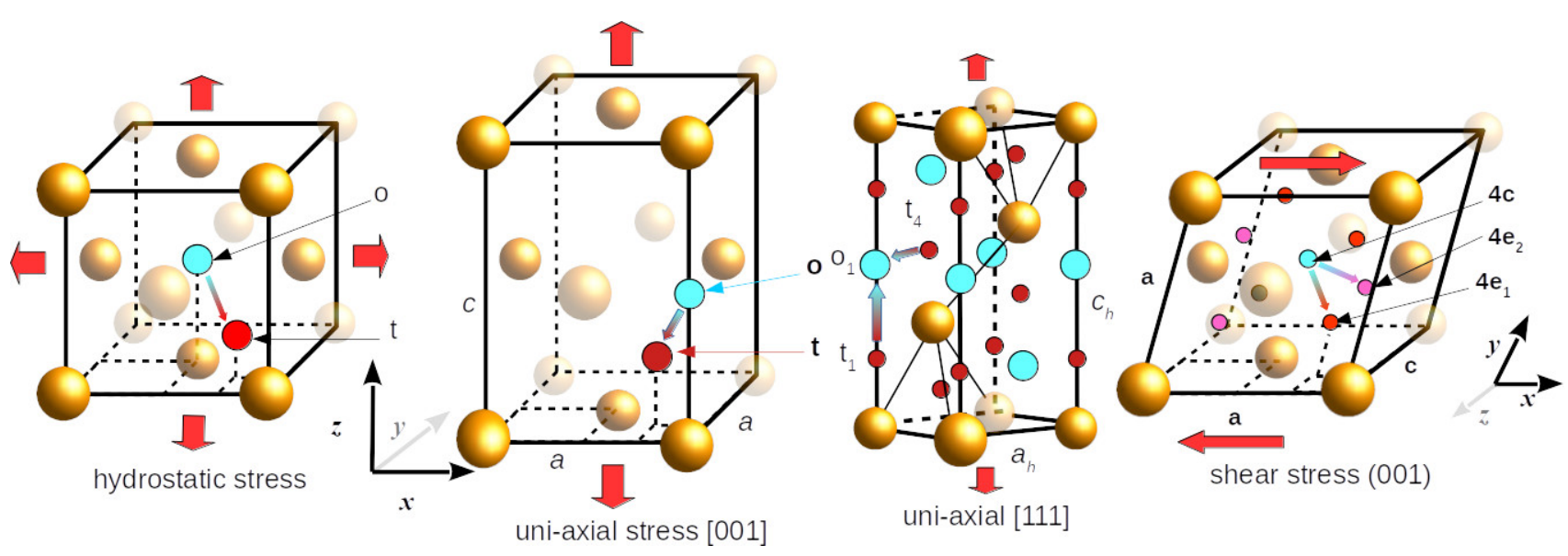

Figure 1. Detail of atomistic jumps for each loading. From left to right: hydrostatic, uni-axial [001], uni-axial [111] and shear (001) loading. Yellow spheres represent atoms of the fcc network, blue and red/pink spheres are the octahedral and tetrahedral hydrogen sites, respectively.

position, i.e. $x$ (either the $o$ or $t$ site). $\mathcal{Z}$ is linked to the vibrational Gibbs energy (labeled $v$ ) through:

$$
G_{v}^{x}=-k_{B} T \ln \mathcal{Z}_{x}
$$

given by

$G_{v}^{x}=k_{B} T \int \sum_{\nu=1}^{3 N} \sum_{\mathbf{q} \in B Z} \delta\left(\omega-\omega_{\nu}^{x}(\mathbf{q})\right) \ln \left[2 \sinh \left(\frac{\hbar \omega}{2 k_{B} T}\right)\right] d \omega$, where $\omega_{\nu}^{x}(\mathbf{q})$ are the (real) frequencies in the wave vector $\mathbf{q}$ and the mode $\nu, \Delta G_{v}^{x y}$, the Gibbs energy difference between the transition state, $t s$, and the initial state, $x$, is defined by:

$$
\Delta G_{v}^{x y}=G_{v}^{t s}-G_{v}^{x} .
$$

In what follows, the effect of loading on $\Delta G_{v}^{x y}$ was studied in the hydrostatic case only.

The direct jumps between two first-nearest neighboring 
octahedral sites $(1 \mathrm{NN}-o)$ and between two first-nearest neighboring tetrahedral $(1 \mathrm{NN}-t)$ sites were omitted for all loadings considered here. The two transition states are actually located in the same place. It is therefore a second-order transition state, and an analysis of its inter-atomic force constants (IFCs) shows two imaginary frequencies. These jumps can then be neglected even at high temperatures, see discussion in Ref. [16]. In the present work, quantum tunnel effects [24, 25] were also neglected for simplification purposes, but could be added. As a first-order approximation, the thermal expansion and anharmonicity were also neglected in this work.

\section{B. Tri-axial loading}

The next case covers a loading in three directions with different amplitudes, $\left\{\sigma_{i i}\right\}_{i=x, y, z}$. The system belongs to the orthorhombic space group No69, Fmmm. As in the previous case, the primitive cell still contains one $o$ site $(4 b)$ and one $t$ site $(8 f)$, see Table I. The symmetry break in the diffusion coefficient is due to the split in lattice parameters, since the only jump considered between first-nearest neighboring $o$ - $t$ sites is along the diagonal of the orthorhombic lattice. The diffusion coefficients still depend on $\Gamma_{t o}$ and $\Gamma_{o t}$ (which depend on $\left\{\sigma_{i i}\right\}$ ) and express as follows:

$$
D_{i}=\frac{a_{i}^{2}}{2} \frac{\Gamma_{o t} \Gamma_{t o}}{\Gamma_{t o}+2 \Gamma_{o t}}
$$

The anisotropy associated with the diffusivity $\left(A_{x-y}=\right.$ $D_{y} / D_{x}$, for example) is thus a function of $\sigma_{i i}$ through its dependence on lattice parameters $\{a, b, c\} . A_{i}$ can be approximated in terms of the shear modulus, $\mathcal{C}^{\prime}$ and $\sigma_{i i}$ :

$$
\left\{\begin{array}{l}
A_{x-y}=\left[\frac{b}{a}\right]^{2} \simeq 1+\frac{\sigma_{y y}-\sigma_{x x}}{\mathcal{C}^{\prime}} \\
A_{y-z}=\left[\frac{c}{b}\right]^{2} \simeq 1+\frac{\sigma_{z z}-\sigma_{y y}}{\mathcal{C}^{\prime}} \\
A_{z-x}=\left[\frac{a}{c}\right]^{2} \simeq 1+\frac{\sigma_{x x}-\sigma_{z z}}{\mathcal{C}^{\prime}}
\end{array}\right.
$$

The effect on the symmetry break is therefore solely geometrical, the $A_{i}$ do not depend on the interstitial species in a first-order approximation (in the $o$ and $t$ sites, the deformation induced by hydrogen is isotropic), but depends on the stiffness of the metal only.

From these general equations, relations in the case uniaxial (along the $z$ direction, for instance) or bi-axial loadings (along $x$ and $y$ simultaneously, with the same magnitude, i.e. $[100]+[010])$ can be deduced. In these cases, the fcc system evolves into a tetragonal structure. The system thus belongs to space group 139, I4/ $\mathrm{mmm}$ ( $t I 2$ prototype).

In the case of a uni-axial [001] loading, $\sigma_{z z}$, according to the $x, y$ and $z$ directions, the corresponding diffusion coefficients are:

$$
\left[\begin{array}{c}
D_{x, y}\left(\sigma_{z z}\right)=\frac{a^{2}}{2} \frac{\Gamma_{o t} \Gamma_{t o}}{\Gamma_{t o}+2 \Gamma_{o t}} \\
D_{z}\left(\sigma_{z z}\right)=\frac{c^{2}}{2} \frac{\Gamma_{o t} \Gamma_{t o}}{\Gamma_{t o}+2 \Gamma_{o t}}
\end{array}\right.
$$

$a$ and $c$ are lattice parameters in the $x$ (or $y$ ) and $z$ directions, respectively. All quantities depend on the applied stress $\sigma_{z z}$. The anisotropy can therefore be expressed as a function of the shear modulus, $\mathcal{C}^{\prime}$, and $\sigma_{z z}$ :

$$
A_{001} \simeq 1+\frac{\sigma_{z z}}{\mathcal{C}^{\prime}}
$$

Equations in the case of a bi-axial stress can be easily deduced from these equations by replacing the loading along [001] with a loading along $[100]+[010]$ directions.

\section{Uni-axial [111] loading}

The following case concerns a uni-axial loading along the [111] direction, i.e. perpendicular to the dense planes. The fcc structure can then be viewed as a structure belonging to space group No166, $R \overline{3} m$. Two representations [40] are possible: either with hexagonal symmetry or with rhombohedral symmetry. For simplification purposes, the first representation was chosen.

The lattice characterized by its two parameters $a_{h}$ and $c_{h}$, will be stressed along the $c_{h}$ axis, as shown in Fig. 1 . In this new space group, the number of equivalent $t$ and $o$ sites per $\mathrm{X}$ atom is still two and one, respectively, see Table I. The octahedral sites are symmetrically located in the $3 b$ position, while there is now one degree of freedom for the tetrahedral sites, $z_{h}$, located in the $6 c$ Wyckoff positions. In the present case, $z_{h}$ was set to 0.5. Tests (displacements and phonon) were performed to confirm this value.

To move an atom from an $o$ site to the nearest neighboring $t$ site, there are now two distinctive atomic jumps, as illustrated in Fig. 1: along the $o_{1}-t_{1}$ direction, associated with frequencies $\Gamma_{o_{1} t_{1}}$ and $\Gamma_{t_{1} o_{1}}$, and along $o_{1}-t_{4}$, with $\Gamma_{o_{1} t_{4}}$ and $\Gamma_{t_{4} o_{1}}$. When the ratio $c_{h} / a_{h}$ is equal to $\sqrt{6}$, fcc symmetry is restored, so the two jumps become equivalent. Their new expressions are (here $X, Y$ and $Z$ refer to the axis of the new representation, $X$ and $Y$ in the basal plane and $Z$ along the $c_{h}$ direction):

$$
\left[\begin{array}{c}
D_{X, Y}=\frac{2 a_{h}^{2}}{3} \frac{\Gamma_{o_{1} t_{4}} \Gamma_{t_{1} o_{1}}+\Gamma_{o_{1} t_{1}} \Gamma_{t_{4} o_{1}}+6 \Gamma_{o_{1} t_{4}} \Gamma_{t_{4} o_{1}}}{2 \Gamma_{o_{1} t_{1}}+6 \Gamma_{o_{1} t_{4}}+\Gamma_{t_{1} o_{1}}+3 \Gamma_{t_{4} o_{1}}} \\
D_{Z}=\frac{c_{h}^{2}}{3} \frac{\Gamma_{o_{1} t_{4}} \Gamma_{t_{1} o_{1}}+\Gamma_{o_{1} t_{1}} \Gamma_{t_{4} o_{1}}}{2 \Gamma_{o_{1} t_{1}}+6 \Gamma_{o_{1} t_{4}}+\Gamma_{t_{1} o_{1}}+3 \Gamma_{t_{4} o_{1}}}
\end{array}\right.
$$

The anisotropy of diffusion, $A_{111}[\sigma]=\frac{D_{Z}}{D_{X, Y}}$, is thus expressed as:

$$
A_{111}=\frac{1}{2}\left[\frac{c_{h}}{a_{h}}\right]^{2} \frac{\Gamma_{o_{1} t_{4}} \Gamma_{t_{1} o_{1}}+\Gamma_{o_{1} t_{1}} \Gamma_{t_{4} o_{1}}}{\Gamma_{o_{1} t_{4}} \Gamma_{t_{1} o_{1}}+\Gamma_{o_{1} t_{1}} \Gamma_{t_{4} o_{1}}+6 \Gamma_{o_{1} t_{4}} \Gamma_{t_{4} o_{1}}}
$$

Here, the anisotropy depends not only on lattice parameters but also on jump rates. As a first-order approximation, the effect of geometric effect can be expressed as (cf. Eq. 23 below):

$$
\left[\frac{c_{h}}{a_{h}}\right]^{2} \simeq 6\left(1+\frac{\sigma_{111}}{\mathcal{C}_{44}}\right)
$$




\section{Shear loading}

Under shear loading, the cubic symmetry evolves into a monoclinic space group 15 (C2/c). We considered a pure shear loading in the $x y$ plane, resulting in a change in the angle $\gamma$ between axes $x$ and $y$. Unit-cell parameters and Wyckoff positions are summarized in Table I. There is still one octahedral site (in the Wyckoff position $4 \mathrm{c}$ ) but there are now two non-equivalent tetrahedral sites, both in the $4 e$ Wyckoff position, labeled $4 e_{1}$ and $4 e_{2}$. Four jumps are therefore necessary to describe atomic jumps. For example, between sites $4 c$ and $4 e_{1}$ (resp. $4 e_{2}$ ) there are two distinct jumps, a short one $(S)$ and a long one $(L)$, written $\Gamma^{S}$ and $\Gamma^{L}$, respectively. The general equations, that include all degrees of freedom of the two $t$ sites, are complex (a total of 8 jump rates). When considering the special case $y_{e_{1}}=0.5$ and $y_{e_{2}}=0.0$, which corresponds to $\mathrm{H}$ atoms occupying $t$ sites in the middle of the "tetrahedral volumes" diffusion coefficients can be simplified as follows:

$$
\begin{aligned}
D_{x, y} & =\frac{a^{2}}{4} \frac{\left(\Gamma_{o t}^{L}+\Gamma_{o t}^{S}\right)\left(\Gamma_{t o}^{L}+\Gamma_{t o}^{S}\right)}{2 \Gamma_{o t}^{L}+2 \Gamma_{o t}^{S}+\Gamma_{t o}^{L}+\Gamma_{t o}^{S}} \\
D_{z} & =\frac{c^{2}}{2} \frac{\Gamma_{o t}^{S} \Gamma_{t o}^{L}+\Gamma_{o t}^{L} \Gamma_{t o}^{S}}{2 \Gamma_{o t}^{L}+2 \Gamma_{o t}^{S}+\Gamma_{t o}^{L}+\Gamma_{t o}^{S}}
\end{aligned}
$$

Here, the reasonable hypothesis is that $e_{1}$ and $e_{2}$ sites give the same jump rates, i.e. $\Gamma_{o t_{1}}^{L}=\Gamma_{o t_{2}}^{L}$.

The anisotropy coefficient, $A_{s}=D_{z} / D_{x, y}$, can be thus determined (as a function of the $\gamma$ angle):

$$
A_{s}[\gamma]=2\left[\frac{c}{a}\right]^{2} \frac{\Gamma_{o t}^{S} \Gamma_{t o}^{L}+\Gamma_{o t}^{L} \Gamma_{t o}^{S}}{\left(\Gamma_{o t}^{L}+\Gamma_{o t}^{S}\right)\left(\Gamma_{t o}^{L}+\Gamma_{t o}^{S}\right)}
$$

In the absence of shear stress, i.e. when $\gamma=\pi / 2, \Gamma_{t o}^{L}=$ $\Gamma_{\text {to }}^{S}$ and $\Gamma_{o t}^{L}=\Gamma_{o t}^{S}, A_{s}$ is thus equal to one.

\section{ELASTICITY THEORY}

The elasticity theory succeeds in describing how applied stresses affect the enthalpies involved in the solubility and diffusivity of point defects in a crystal [45-47]. Several works already use this approach to study the diffusion, the solubility of point defects or their interactions with extended defects. [38, 48-51]. This requires only a limited number of parameters, which can be obtained beforehand through DFT calculations. The validity of this elastic theory approach is limited to the domain where the relationship between stress and strain is linear (i.e. low magnitude stresses). However, the validity remains excellent for large stress magnitudes as seen below.

When theoretically studying the effect of a mechanical loading, one can either set the strain tensor to a fixed value (strain control) or, alternatively, set the stress tensor (stress control). The latter option best describes real mechanical tests applied to a single crystal, and has been chosen here. In this case, the stress dependency of the enthalpy functions that describe diffusion thermodynamics can be calculated [32, 39].

\section{A. Solubility}

To study the effect of a stress tensor $\boldsymbol{\sigma}$ applied to a crystal on the insertion enthalpy of hydrogen, the hydrogen-stress interaction energy was taken into account via (in Einstein's notation):

$$
H_{\mathrm{ins}}^{s}(\boldsymbol{\sigma})=U_{\mathrm{ins}}^{s}(0)-\mathcal{V}_{i j}^{s} \sigma_{i j}
$$

$U_{\text {ins }}^{s}(0)$ is the insertion energy of hydrogen in site $s(t$ or $o)$ in the absence of stress. $\mathcal{V}^{s}$ is the relaxation volume tensor of hydrogen in site $s$. It is related to the elastic dipole tensor $\mathcal{P}^{s}$ through:

$$
\mathcal{V}_{i j}^{s}=\mathcal{S}_{i j k l} \mathcal{P}_{k l}^{s}
$$

where $\mathcal{S}$ is the elastic compliance tensor of the crystal, inverse of the stiffness tensor $\mathcal{C}$. Components of $\mathcal{P}^{s}$ and $\mathcal{C}$ were computed using DFT calculations, see Tables II and III.

\section{B. Diffusivity}

To take into account the applied stress in the migration mechanism, the migration enthalpy from $o$ to $t$ was written:

$$
H_{\mathrm{m}}^{o-t}(\boldsymbol{\sigma})=U_{\mathrm{m}}^{o-t}(0)-\left(\mathcal{V}_{i j}^{t s}-\mathcal{V}_{i j}^{o}\right) \sigma_{i j}
$$

The difference $\Delta \mathcal{V}^{o}=\mathcal{V}^{t s}-\mathcal{V}^{o}$ between the transition state $t s$ and the initial position $o$ is the migration volume tensor. It is related to the migration dipole tensor through $\Delta \mathcal{V}_{i j}^{o}=\mathcal{S}_{i j k l} \Delta \mathcal{P}_{k l}^{o}$. $U_{\mathrm{m}}^{o-t}(0)$ is the zero-stress case migration energy, it is equal to

$$
U_{\mathrm{m}}^{o-t}(0)=U_{\mathrm{ins}}^{t s}(0)-U_{\mathrm{ins}}^{o}(0) .
$$

For the migration enthalpy from $t$ to $o, H_{\mathrm{m}}^{t-o}(\boldsymbol{\sigma})$, corresponding equations can be obtained similarly.

\section{Effect on lattice parameters}

As shown above, the symmetry break of the hydrogen diffusion coefficient is partly due to the symmetry break in the lattice parameters. Elasticity can capture this evolution with the strain-stress relationship

$$
\varepsilon_{i j}=\mathcal{S}_{i j k l}\left(\sigma_{k l}+\mathcal{P}_{k l} / V\right) .
$$

In this equation, the first term is the strain response to the applied stress tensor, while the second term is the homogeneous strain induced by the interstitial $\mathrm{H}$ atom present in the DFT super-cell of volume $V$. From Eq. 
21, the lattice parameters of the conventional cell can be extracted via $a_{i}=a_{0}\left(1+\varepsilon_{i i}\right)$, where $a_{0}$ is the lattice parameter of the stress-free hydrogen-free crystal.

In the case of uni-, di- and tri-axial loadings, lattice parameters are written as a function of the normal stress components $\sigma_{i i}$ :

$$
\left\{\begin{array}{l}
a=a_{0}\left(1+\frac{\left(\mathcal{C}_{11}+\mathcal{C}_{12}\right) \sigma_{11}-\mathcal{C}_{12}\left(\sigma_{22}+\sigma_{33}\right)}{6 B \mathcal{C}^{\prime}}+\frac{\mathcal{P}_{11}}{3 B V}\right. \\
b=a_{0}\left(1+\frac{\left(\mathcal{C}_{11}+\mathcal{C}_{12}\right) \sigma_{22}-\mathcal{C}_{12}\left(\sigma_{11}+\sigma_{33}\right)}{6 B \mathcal{C}^{\prime}}+\frac{\mathcal{P}_{11}}{3 B V}\right. \\
c=a_{0}\left(1+\frac{\left(\mathcal{C}_{11}+\mathcal{C}_{12}\right) \sigma_{33}-\mathcal{C}_{12}\left(\sigma_{11}+\sigma_{22}\right)}{6 B \mathcal{C}^{\prime}}+\frac{\mathcal{P}_{11}}{3 B V}\right.
\end{array}\right)
$$

$\mathcal{C}_{i j}$ are the stiffness constants of the crystal, $B=\left(\mathcal{C}_{11}+\right.$ $\left.2 \mathcal{C}_{12}\right) / 3$ is the bulk modulus and $\mathcal{C}^{\prime}=\left(\mathcal{C}_{11}-\mathcal{C}_{12}\right) / 2$ is the shear modulus.

Similarly, the lattice parameters of the hexagonal cell are given as a function of the stress $\sigma_{111}$ applied along direction [111] of the conventional cell (i.e. direction [0001] of the hexagonal cell):

$$
\left\{\begin{aligned}
a_{h}\left[\sigma_{111}\right] & =\frac{\sqrt{2}}{2} a_{0}\left(1+\frac{1}{3}\left(\frac{1}{3 B}-\frac{1}{2 \mathcal{C}_{44}}\right) \sigma_{111}+\frac{\mathcal{P}_{11}}{3 B V}\right) \\
c_{h}\left[\sigma_{111}\right] & =\sqrt{3} a_{0}\left(1+\frac{1}{3}\left(\frac{1}{3 B}+\frac{1}{\mathcal{C}_{44}}\right) \sigma_{111}+\frac{\mathcal{P}_{11}}{3 B V}\right)
\end{aligned}\right.
$$

In the case of a pure in-plane shear stress in plane (001), the lattice parameters are uniformly modified by the $\mathrm{H}$ insertion, but remain insensitive to the applied shear stress:

$$
a=b=c=a_{0}\left(1+\frac{\mathcal{P}_{11}}{3 B V}\right) .
$$

\section{DFT APPROACH}

First-principles calculations were carried out, to be confronted with the elasticity theory, to identify limitations, and compute elementary parameters presented above. Insertion and migration energies were computed using the Vienna $a b$ initio simulation package (VASP) [52]. The self-consistent Kohn-Sham equations were solved using the projector augmented wave (PAW) pseudo-potentials [53, 54], and the PerdewBurke-Ernzerhof (PBE [55]) exchange and correlation functional. The plane-wave energy cut-off was set to 600 $\mathrm{eV}$. Calculations were conducted on $2 \times 2 \times 2$ super-cells, i.e. 32 atoms, except those for the uni-axial [111] loading (hexagonal $3 \times 3 \times 2$ super-cells, i.e. 54 atoms) and those executed to obtain the data listed in Table III. In the first super-cell, the Brillouin zone was sampled by $\Gamma$-centered $12 \times 12 \times 12$ Monkhorst-Pack meshes [56]. Tests were carried out with finer grids $24 \times 24 \times 24$. Compared results show that the difference is small, under $1 \%$. Some tests were also performed to evaluate the effect of the size of the supercells used: thus some calculations were made on larger supercells (3x3x3, i.e. 108 atoms per unit cell) in the case of hydrostatic deformation where the effects are the most pronounced (see below). The results show a weak dependence of the effect of the cell used. The lattice and ion relaxations were carried out by using a conjugate-gradient algorithm.

The components of the stress tensor were chosen and fixed depending on the loading considered. Atomic forces were minimized to a value below $0.005 \mathrm{eV} / \AA$. For example, in the case of a uni-axial loading in direction [001], different values of stress $\sigma_{z z}$ were imposed, and the other components of the stress tensor were set to zero. At the end of the simulations, a set of DFT energies and lattice parameters were obtained and fit according to $\sigma_{z z}$.

The insertion enthalpy in a $s$ site, $G_{\text {ins }}^{s}$, thus writes:

$$
G_{\mathrm{ins}}^{s}(\boldsymbol{\sigma}, T)=U_{\mathrm{ins}}^{s}(\boldsymbol{\sigma}, T)+G_{\mathrm{ins}, v}^{s}(\boldsymbol{\sigma}, T)-V \sigma_{i j} \epsilon_{i j}^{\mathrm{rel}}
$$

where $U_{\text {ins }}^{s}$ is the internal energy, $G_{\text {ins }, v}^{s}$ the vibrational Gibbs energy of insertion, $V$ the volume of the super-cell and $\epsilon_{i j}^{\text {rel }}$ the uniform strain resulting from relaxation. All quantities were computed for the same loading. $U_{\text {ins }}^{s}$ can then be expressed in terms of DFT energies, $E_{0}$, with and without hydrogen in $s$ and hydrogen energy of the $\mathrm{H}_{2}$ molecule, $E_{0}\left[\mathrm{H}_{2}\right]$, as:

$$
\begin{array}{r}
U_{\mathrm{ins}}^{s}(\boldsymbol{\sigma}, T)=E_{0}^{\mathrm{bulk}+\mathrm{H}}(\boldsymbol{\sigma}, T)-E_{0}^{\mathrm{bulk}}(\boldsymbol{\sigma}, T) \\
-\frac{1}{2} E_{0}\left[\mathrm{H}_{2}\right]
\end{array}
$$

$G_{\mathrm{ins}, v}^{s}$ is given by:

$$
\begin{array}{r}
G_{\mathrm{ins}, v}^{s}(\boldsymbol{\sigma}, T)=G_{v}^{\mathrm{bulk}+\mathrm{H}}(\boldsymbol{\sigma}, T)-G_{v}^{\mathrm{bulk}}(\boldsymbol{\sigma}, T) \\
-\frac{1}{2} \mu\left[\mathrm{H}_{2}\right](T)
\end{array}
$$

with $G_{v}$, the Gibbs energy of the bulk with and without hydrogen, and $\mu\left[H_{2}\right](T)$ the chemical potential of $\mathrm{H}_{2}$ in the equilibrated gas. The chemical potential writes as:

$$
\begin{array}{r}
\mu\left[H_{2}\right](T)=\frac{1}{2} \hbar \omega\left[H_{2}\right]-k_{B} T\left[\frac{3}{2} \ln \left(\frac{T}{A_{t}}\right)+\ln \left(\frac{k_{B} T}{p_{o}}\right)\right. \\
\left.-\ln \left(1-e^{-\Theta_{v i b} / T}\right)+\ln \left(\frac{T}{\sigma \Theta_{\text {rot }}}\right)+\ln \left(q_{e}\right)+1\right]
\end{array}
$$

where $A_{t}=h^{2} / 2 \pi m k_{B}, \Theta_{v i b}=h \nu / k_{B}, \Theta_{\text {rot }}=$ $\hbar^{2} / 2 I_{r} k_{B}, m=2 M$ ( $M$ atomic mass) and $I_{r}=$ $M d\left(H_{2}\right)^{2} / 2$ the moment of inertia of $\mathrm{H}_{2}$. The first term corresponds to the zero-point energy, the second to the translational components of the partition function of the $\left(H_{2}\right)$ molecule, $\ln \left(1-e^{-\Theta_{v} / T}\right)$ to the vibrational component, $\ln \left(T / \sigma \Theta_{r}\right)$ to the rotational one and $q_{e}$ to the electronic component. The parameters used in the present work are: $\omega\left[H_{2}\right]=540 \mathrm{meV}, A_{t}=15110^{-20} \mathrm{~K} \cdot \mathrm{m}^{2}$, $\Theta_{v}=6266 \mathrm{~K}(6331 \mathrm{~K}[57]), \Theta_{r}=85.5 \mathrm{~K}(87.6 \mathrm{~K}$ [57]), $I_{r}=0.04710^{-46} \mathrm{~kg} \cdot \mathrm{m}^{2}, d\left(\mathrm{H}_{2}\right)=0.75 \AA, \sigma=2$ and $q_{e}=1$. The regular formula [57] contains a term that depends explicitly on partial pressure $\mathrm{H}_{2}, p_{\mathrm{H}_{2}}$, i.e. $+k_{B} T \ln \left(p_{H_{2}}\right)$. Here, it was already included in the prefactor of the concentration formula, Eq. 1 hence the term $\sqrt{p_{H_{2}} / p_{o}}$. 
To study migration processes, CLIMB-NEB simulations [58] were conducted on $2 \times 2 \times 2$ super-cells with the same stress. The energy of the transition state was then computed with a $600 \mathrm{eV}$ energy cut-off and $12 \times 12 \times 12 \mathrm{k}$ meshes to sample the first-Brillouin zone. The phonopy package [59] was then used to compute the inter-atomic force constants (IFCs) of stable and transition states. Gibbs energies, given in Eq. 5, were then computed using $20 \times 20 \times 20$ q-meshes.

\section{PRELIMINARY RESULTS}

To use the theory of elasticity, different parameters calculated at zero pressure are required: the insertion energy, $E_{\text {ins }}$, the migration energies, $E_{\mathrm{m}}$, the attempt frequencies, $\Gamma_{o t}$ and $\Gamma_{t o}$, the lattice parameter, $a_{0}$, the elastic constants, $\mathcal{C}$, and the elastic dipoles, $\mathcal{P}$ for each site, i.e. $o$ and $t$ sites, and when the $\mathrm{H}$ atom is in the transition state. In this section, these various parameters are presented and discussed.

\section{A. Ground-state parameters of phases}

Data on the four fcc systems (Al, $\mathrm{Ni}, \mathrm{Cu}$ and $\mathrm{Pd}$ ) are gathered in Table II, i.e. lattice parameters, $a_{0}$, and elastic constants (calculated using DFT on the primitive cell), $\mathcal{C}_{i j}$.

Table II. Lattice parameters, $a_{0}$ in $\AA$, and elastic constants, $\mathcal{C}_{i j}$ in $\mathrm{GPa}$, of $\mathrm{Al}, \mathrm{Ni}, \mathrm{Cu}$ and $\mathrm{Pd}$ systems. The first number is the value calculated in this work, the second number corresponds to experimental values. From these data the bulk modulus, $B=\left(\mathcal{C}_{11}+2 \mathcal{C}_{12}\right) / 3$, and shear modulus, $\mathcal{C}^{\prime}=\left(\mathcal{C}_{11}-\mathcal{C}_{12}\right) / 2$, were also computed.

\begin{tabular}{ccccc}
\hline \hline & $\mathrm{Al}[60]$ & $\mathrm{Ni}[60,61]$ & $\mathrm{Cu}[60,62,63]$ & $\mathrm{Pd}[60,64]$ \\
\hline$a_{0}$ & $4.04 / 4.05$ & $3.52 / 3.52$ & $3.62 / 3.61$ & $3.94 / 3.87$ \\
$\mathcal{C}_{11}$ & $105 / 116$ & $272 / 253$ & $193 / 168-173$ & $202 / 234$ \\
$\mathcal{C}_{44}$ & $33 / 31$ & $125 / 122$ & $85 / 61-76$ & $60 / 71$ \\
$\mathcal{C}_{12}$ & $65 / 65$ & $158 / 158$ & $134 / 118-129$ & $153 / 176$ \\
$\mathcal{C}^{\prime}$ & $20 / 25$ & $57 / 47$ & $29 / 24$ & $25 / 29$ \\
$B$ & $78 / 82$ & $196 / 189$ & $154 / 138$ & $169 / 181$ \\
\hline \hline
\end{tabular}

DFT results are found in excellent agreement with experimental and theoretical values [60-64]. These DFT values will be used in the remainder of this work, to calculate the compliance tensor, $\mathcal{S}$.

\section{B. Stress-free DFT parameters}

Results on hydrogen insertion and migration will now be discussed. Parameters for $\mathrm{H}$ atoms were obtained on large super-cells (up to $6 \times 6 \times 6$ primitive cells, i.e. 216 atoms, for $\mathrm{Al}$, and $3 \times 3 \times 3$ cubic cells for the other systems), they are summarized in Table III. To quantify the effect of the size of the super-cell used, values corresponding to intermediate-size super-cells are also reported.

At zero-pressure, results show that in the Al system, $\mathrm{H}$ atoms occupy preferentially $t$ sites in agreement with literature findings $[13,23]$, whereas in $\mathrm{Ni}[13,16,23], \mathrm{Cu}$ $[13,16,23]$ and $\operatorname{Pd}[13,16,22]$ systems, $\mathrm{H}$ atoms prefer $o$ sites. In the case of $\mathrm{Al}$ and $\mathrm{Pd}$, the difference between $o$ and $t$ sites is low, less than $150 \mathrm{meV}$. In all configurations, zero-point energies (ZPEs) were evaluated from the IFCs, values are given in Table III. These results also confirm that both sites are stable for all systems. On the other hand, ZPE values are low and do not change the relative stability of $t$ and $o$ sites. In the case of $\mathrm{Al}$, the $\mathrm{ZPE}$ decreases the energy difference between the $o$ and $t$ sites, without modifying their relative stability.

Concerning the migration process, as explained in Section II, at ambient pressure, the main atomic jump is between first-nearest neighboring $o$ and $t$ sites. Indeed, for the two other jumps, i.e. between $1 \mathrm{NN}-o$ and $1 \mathrm{NN}-t$, the transition states are located in the same position, which is therefore a second-order transition state [16]. The energy barriers between $o$ - $t$ sites, $U_{m}$, listed in Table III are equivalent to those found in the theoretical literature $[16,23,42]$ but also in excellent agreement with experimental data: $0.435 \mathrm{eV}$ in $\mathrm{Cu}$ [21], $0.410 \mathrm{eV}$ in $\mathrm{Ni}$ [65] and $0.226 \mathrm{eV}$ in $\mathrm{Pd}$ [66].

From data given in Table III, the values calculated at constant pressure (zero) and at constant volume can also be compared. The results show that, on large supercells, unrelaxed calculations (constant volume calculations) provide accurate results. But the difference between the two approaches is always small whichever the super-cell used. This is specific to hydrogen. It comes from the fact that inserted hydrogen atoms generate small local distortions. Indeed, the values of the components of the elastic tensors are low. For other interstitial species ( $\mathrm{C}, \mathrm{N}$ or $\mathrm{O}$ ), this effect should be stronger.

In the rest of the study, these insertion and migration energy values will be considered as the stress-free reference parameters.

In addition to these parameters, new data are listed in Table III: elastic dipoles, $\mathcal{P}$, relaxation volume tensor, $\mathcal{V}$, interaction energies with periodic images, $E_{\mathrm{p}}$, and volumes of relaxation, $V_{\text {rel }}$. They quantify the elastic effects of the insertion. They were calculated either within the framework of the elasticity theory or directly from DFT simulations. The elastic and relaxation volume tensors were obtained using the Varvenne method [36] from the DFT values of the residual stress for the calculations performed at fixed volume (that of the perfect system) and from the elastic constants $\mathcal{C}$ of the metal given in Table II. $E_{\mathrm{p}}$, the correction energy due to the periodic images is as low as a few meV for the three configurations (o, $t$ and transition states) even for small super-cells. For both $t$ and $o$ sites, $\mathcal{P}$ and $\mathcal{V}$, are diagonal and conserve the cubic symmetry. For the tensors of the transition 
Table III. Elastic dipole $\left(\mathcal{P}\right.$, in eV), relaxation volume tensor $\left(\mathcal{V}\right.$, in $\left.\AA^{3}\right)$, insertion energy $\left(U_{\text {ins }}\right.$, in meV), relaxation volume $\left(V_{\text {rel }}\right.$, in $\left.\AA^{3}\right)$, migration energy $\left(U_{\mathrm{m}}\right.$, in meV), interaction energy with periodic images $\left(E_{\mathrm{p}}\right.$, in meV) and the zero-point energy (ZPE, in $\mathrm{meV}$ ) of the four fcc systems. The values calculated at either constant volume (labeled $\dagger$ ) or constant pressure (stress-free) are both summarized below.

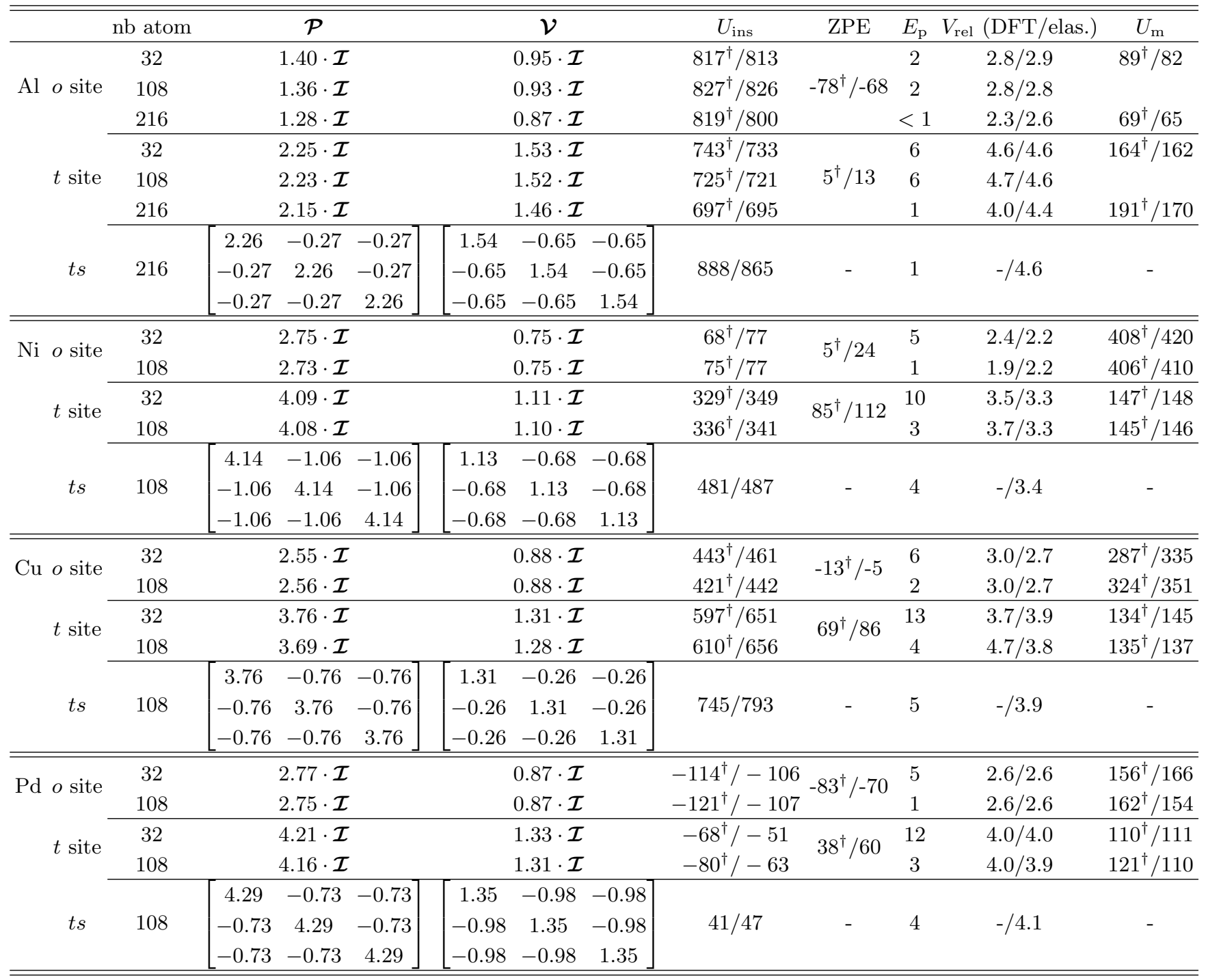

states, the only off-diagonal contribution is small.

The comparison between the insertion in $t$ and $o$ sites shows that $t$ sites induce the strongest elastic effects. These results are consistent with the fact that the free volume of each configuration is smaller in $t$ sites than in $o$ sites. It should also be noted that DFT results are remarkably reproduced by the elasticity theory.

It is interesting to discuss the effect of box size on the results. Indeed, in the case of $\mathrm{Al}$ studied thoroughly below, the DFT results obtained on $2 \times 2 \times 2$ super-cells are compared to elasticity theory results calculated from DFT parameters obtained on larger super-cells, i.e. 216 atoms. The difference between the results obtained from these two cell sizes is relatively low but exist nonetheless. It is about $5-10 \%$ for $\mathrm{Al}$, and is lower for other metals. The analysis will therefore be performed with the $\mathcal{P}_{i j}$ values of the largest boxes.

\section{Elasticity theory results}

The parameters used for the elasticity theory were determined from the values calculated in the framework of the first-principles theory. The Al system was used as the reference system. The transition state relaxation tensor for $\mathrm{H}$ in $\mathrm{Al}$, computed using the elasticity theory from the DFT dipole tensor, Table III in $\AA^{3}$, is equal to:

$$
\mathcal{V}^{\mathrm{ts}}=\left[\begin{array}{ccc}
1.54 & -0.65 & -0.65 \\
-0.65 & 1.54 & -0.65 \\
-0.65 & -0.65 & 1.54
\end{array}\right]
$$


The components of tensor $\mathcal{V}^{\text {ts }}$ computed using elastic dipoles are close to those calculated directly from DFT simulations, see Table III.

From these parameters, a linear correction of the stress effect on insertion and migration enthalpies was calculated using equations summarized in Section III. Results for the different loadings are gathered in Tables IV and $\mathrm{V}$. These results are discussed below.

\section{DISCUSSION}

\section{A. Hydrostatic loading}

Among the different loadings tested, hydrostatic loading produces the strongest effect. From an experimental standpoint, the range of hydrostatic stress has no limit, contrary to others loading. The maximal pressure is only limited by phase transitions. In the case of $\mathrm{Al}$, the insertion in $t$ and $o$ sites and the migration enthalpies of $\mathrm{H}$ between $o$ to $t$ sites (without the zero-point energy), are depicted in Fig. 2 (top) as a function of pressure, $p=-\sigma_{i i} / 3$.

When the system is under compression (resp. tension) i.e. $p>0$ (resp. $p<0$ ), the enthalpy of insertion increases (resp. decreases), see top plot in Fig. 2. The available space for the $\mathrm{H}$ atom is directly related to the applied stress, as illustrated by the evolution of the lattice parameter shown in Fig. $2\left(a_{\bmod }=a_{o}\left(1-\frac{p}{3 B}\right)\right)$. It is therefore more difficult to insert $\mathrm{H}$ into $\mathrm{Al}$ under compression than under tension.

Furthermore, the relative stability of $o$ and $t$ sites under pressure can be analyzed. In the $\mathrm{Al}$ system, $t$ sites are more stable than $o$ sites at ambient pressure, as seen earlier. In Fig. 2 (middle plot), the enthalpies of insertion in $t$ and $o$ sites are also represented taking into account the ZPE values given in Table III. The ZPE should show little dependence on $p$, see above. The enthalpy difference between the two configurations increases as $p$ decreases. At negative pressures, the stability of $t$ sites is then maintained: they remain more stable than octahedral sites. However, when the pressure is greater than about $+1 \mathrm{GPa}$, $o$ sites become more stable than $t$ sites. Anticipating the results below, this effect is specific to the $\mathrm{Al}$ system, where pressure switches the relative stability of the sites. In the other fcc systems, the influence of $p$ is too small and the difference between $o$ and $t$ sites is too high to be compensated by $p$.

It can also be noted that below about $-5 \mathrm{GPa}$, the octahedral sites become unstable and three imaginary frequencies appear (not shown here), which has a direct impact on the diffusion coefficient of hydrogen, since the tetrahedral sites are the only stable configurations remaining. Therefore, octahedral sites, with their three imaginary frequencies, are not transition states. For simplification purposes, this aspect was neglected here when discussing diffusion coefficients.

The variation in enthalpy is mainly due to the work
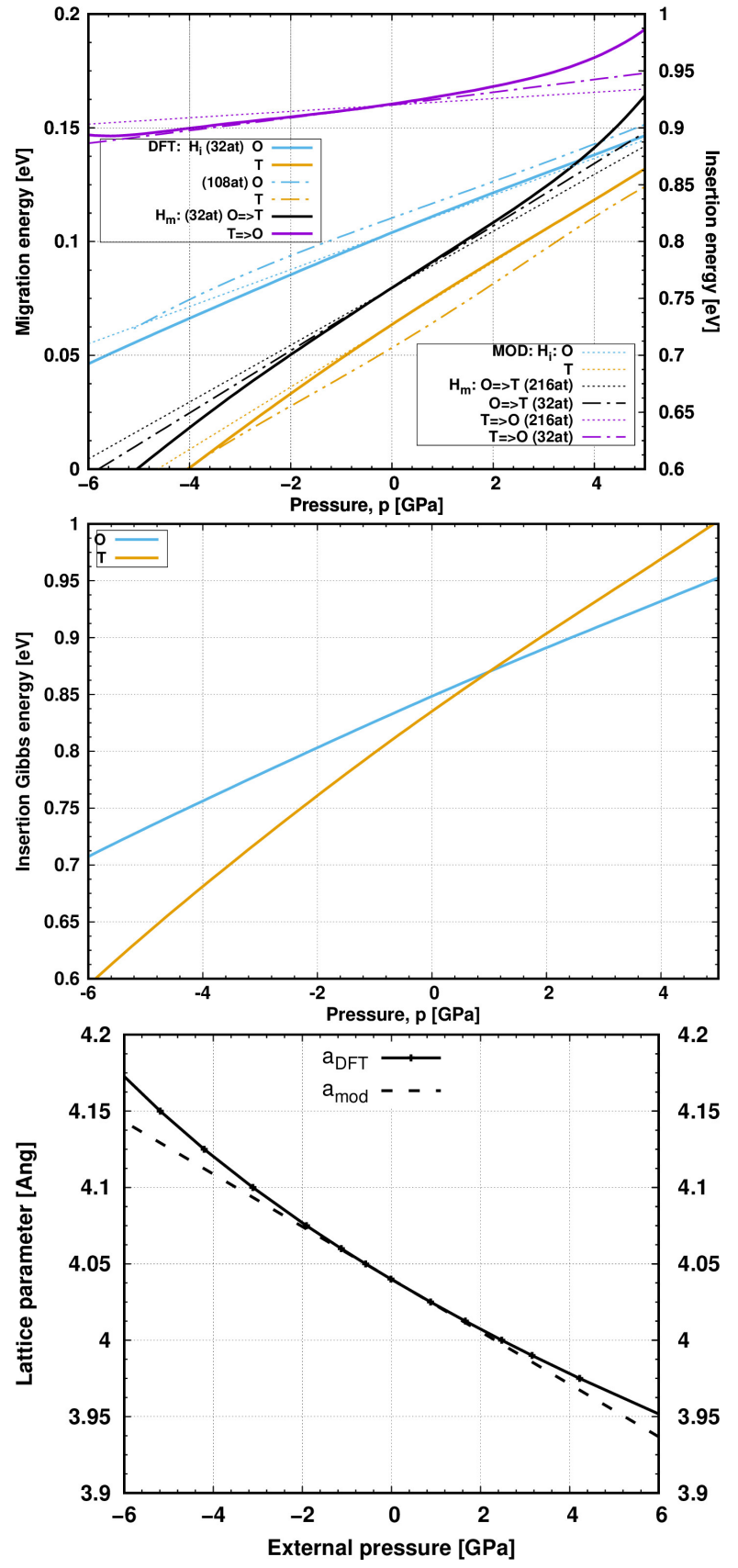

Figure 2. Top plot: formation and migration enthalpies in $\mathrm{Al}$ as a function of hydrostatic pressure $p$, calculated at $0 \mathrm{~K}$ without the ZPE. Two results are presented for the elasticity theory: with the elastic tensor calculated on $2 \times 2 \times 2$ (dashed lines) and on $3 \times 3 \times 3$ super-cells (solid lines). Two results are also presented for DFT results: on $2 \times 2 \times 2$ (solid lines) and on $3 \times 3 \times 3$ super-cells (dashed lines). Middle plot: enthalpies of formation including the ZPE. Bottom plot: lattice parameter evolution in the $\mathrm{Al}$ system as a function of $p$, DFT-elasticity theory comparison, Eq. 22.

of the applied pressure (second term in the right-hand side of Eqs. 17 and 19), because the internal energy does not depend much on the pressure. This observa- 
tion/phenomenon was also confirmed for vacancies [39]. The change in migration enthalpy due to the applied pressure is thus expressed as $\Delta H_{\mathrm{m}}=p \Delta V$ where the activation volume for migration is $\Delta V=\operatorname{tr}(\Delta \mathcal{V})$. For aluminum, values from Table $\mathrm{V}$ lead to $\Delta V^{o-t}=2.01 \AA^{3}$ and $\Delta V^{t-o}=0.23 \AA^{3}$. Hence, the $o-t$ migration barrier is much more sensitive to the applied pressure than the $t$-o migration barrier, see Fig. 2 (top).

As both migration volumes are positive, the enthalpy barriers increase with increasing pressure. It can be inferred that compression slows down hydrogen diffusion (energy barriers increase), whereas under traction, on the contrary, down to $-5 \mathrm{GPa}$, energy barriers decrease quickly. When $p<-5 \mathrm{GPa}$, the migration enthalpy, $H_{\mathrm{m}}$ from $o$ to $t$, becomes negative. This result should be put in perspective with the fact that $o$ sites are unstable under these pressures. For higher pressures, different atomic jumps will have to be considered since this has already been observed in the case of oxygen diffusion in the $\mathrm{Al}$ system [17]. As explained above, DFT simulations were performed on two super-cell sizes: $2 \times 2 \times 2$ and $3 \times 3 \times 3$. Fig. 2 gathers the results. They clearly show that the main effect of the box size is a constant shift of the solubility energy curves. The slopes are however similar. In the case of hydrogen, where the insertion energy is small, the use of small super-cells is therefore sufficiently accurate to capture the physics.

The effect of pressure on the vibrational enthalpy of insertion and migration was then also investigated using DFT simulations. In the case of hydrogen, the vibrational contribution is indeed not negligible. From interatomic forces computed at different pressures, $G_{v}^{s}$ were computed and, results are plotted in Fig. 3. It can be seen that, whichever the applied pressure, the vibrational Gibbs energy of insertion varies slightly, contrary to the vibrational Gibbs energy of migration. In the case of migration, there is a small effect, except in one case (for the jump from $o$ to $t$ ) where a maximum variation of about $50 \mathrm{meV}$ between 0 and $6 \mathrm{GPa}$ is observed at high temperatures. It is reasonable to assume, in first-order approximation, that this will only have a limited effect on the hydrogen diffusion coefficient. As a first approximation, the vibrational effects of pressure will be assumed negligible and neglected. Stress-free vibration enthalpies will therefore be used to compute concentration and the diffusion coefficient.

Elasticity theory and DFT calculations can now be compared. In Fig. 2, the elastic results based on data pooled in Table IV and V are superimposed on the DFT results. The elastic model accurately reproduces the effect of stress when the system is hydrostatically loaded. By examining the results in detail, the effect of pressure on $o$ and $t$ sites is in excellent agreement with the DFT simulations, even quantitatively speaking. The main difference can be found in the slopes of the energy barriers, where values are in lesser good agreement. It should be noted that the values listed in Tables IV and V were obtained using the elastic tensor values taken for $6 \times 6 \times 6$

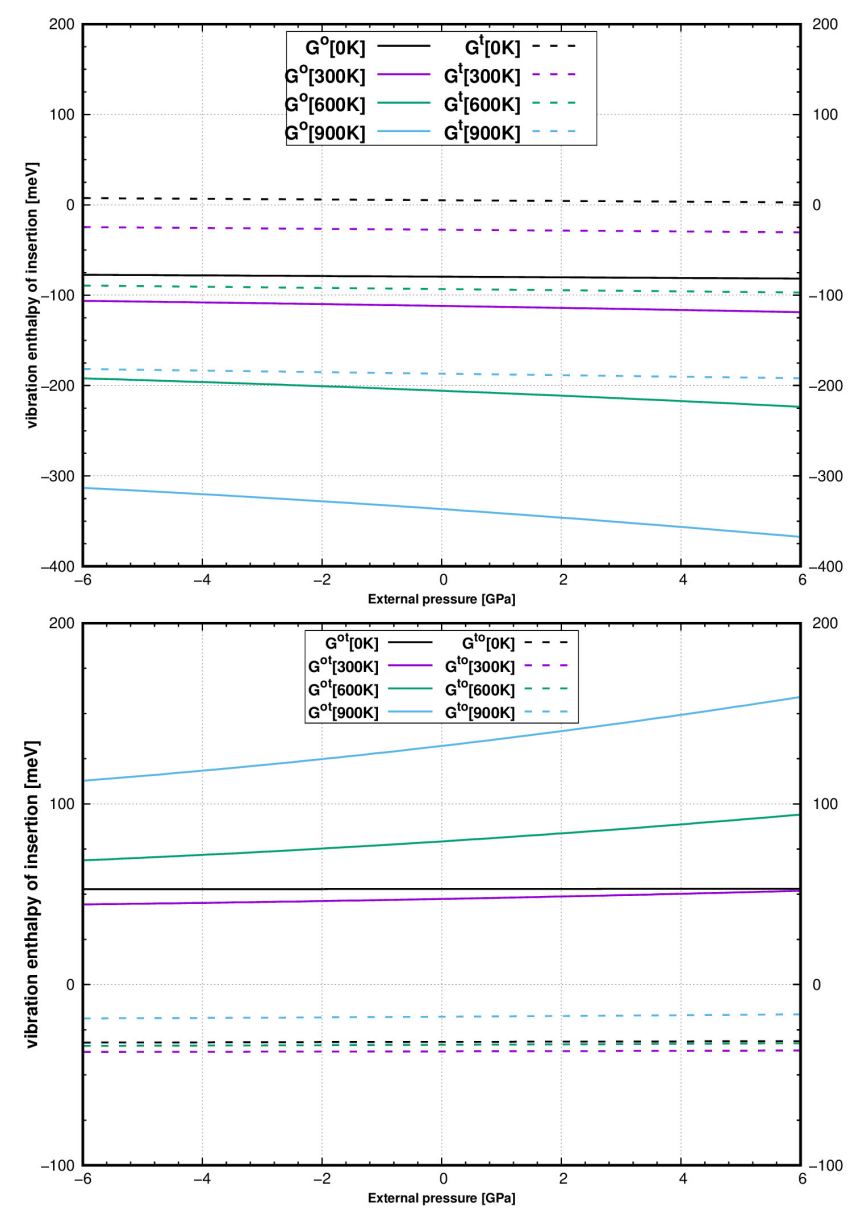

Figure 3. Vibrational formation, $G^{o}$ and $G^{t}$, (top) and migration, $G^{t-o}$ and $G^{o-t}$, (bottom) vibrational Gibbs energy as a function of $p$ (hydrostatic stress), calculated for 4 temperatures: $0,300,600$ and $900 \mathrm{~K}$.

super-cells for $\mathrm{Al}$, whereas the DFT calculations were performed on small super-cells $(2 \times 2 \times 2)$. To quantify the effect of super-cell size, the values of the elastic tensors corresponding to super-cells of the same size $(2 \times 2 \times 2)$ were also used in the elasticity theory. From these new parameters excellent tuning can be obtained, as displayed in Fig. 2 (top plot, dashed lines). This suggests that (i) both approaches (DFT and elasticity theory) are in excellent agreement, (ii) the effect of stress can be reproduced on different types of super-cells, and therefore so can reproduce the effect of concentration, (iii) parameters obtained from large super-cells improve the accuracy of predicted values, and (iv) the validity range of the elasticity theory is obtained until \pm 2 GPa but can be used on larger pressure ranges.

A general overview of other fcc systems can now be obtained from the idea that theory of elasticity can well replace much of the DFT simulations. Data are summarized in Appendix A, Tables IV and V. Compared to Al, it can be noted that in $\mathrm{Ni}, \mathrm{Cu}$ and $\mathrm{Pd}$ systems, the effect of pressure (slopes) is equivalent on hydrogen insertion 

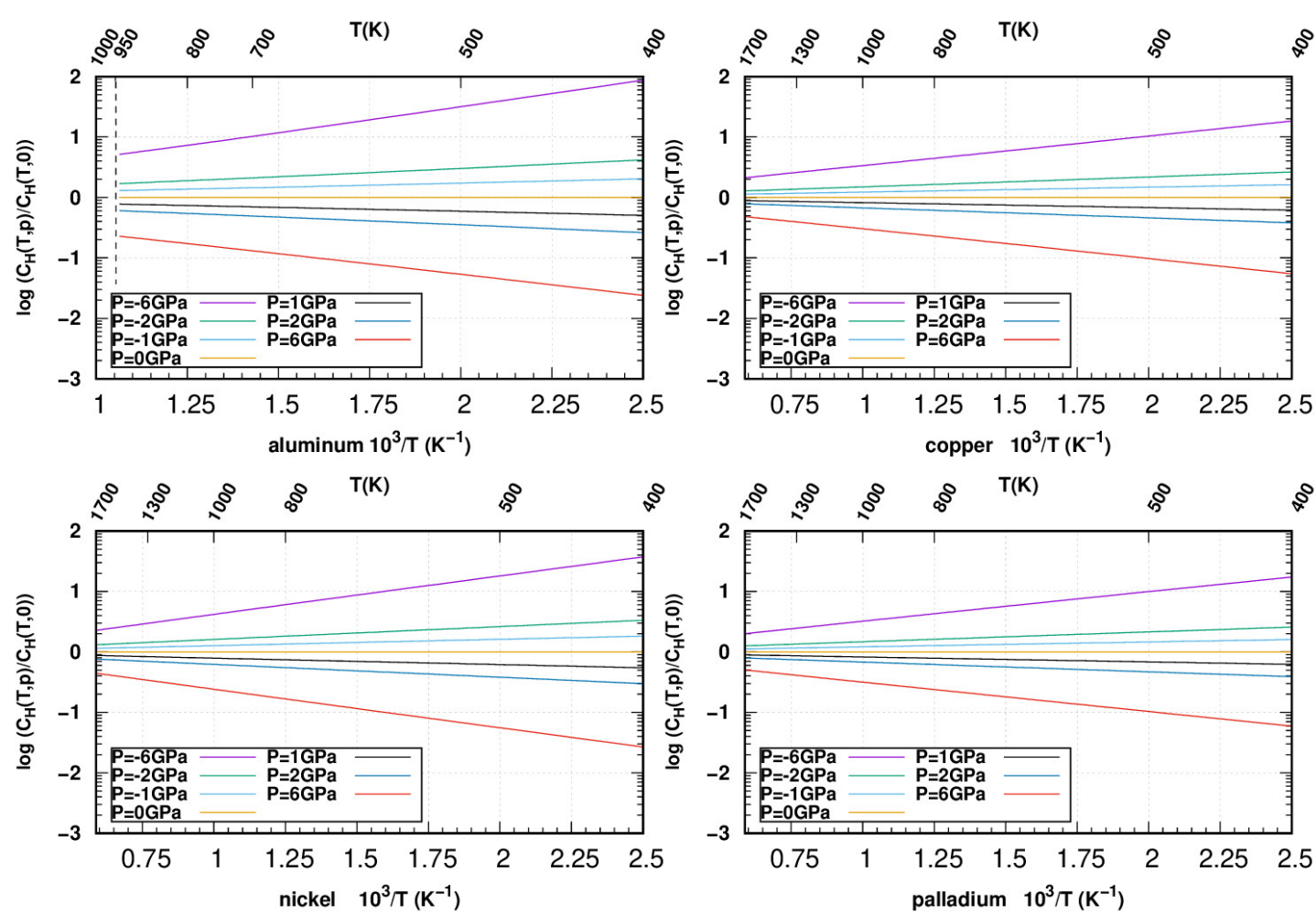

Figure 4. $C_{H}[T, p] / C_{H}[T, 0]$ as a function of temperature under different pressures in $\mathrm{Al}, \mathrm{Cu}, \mathrm{Ni}$ and $\mathrm{Pd}$ systems.
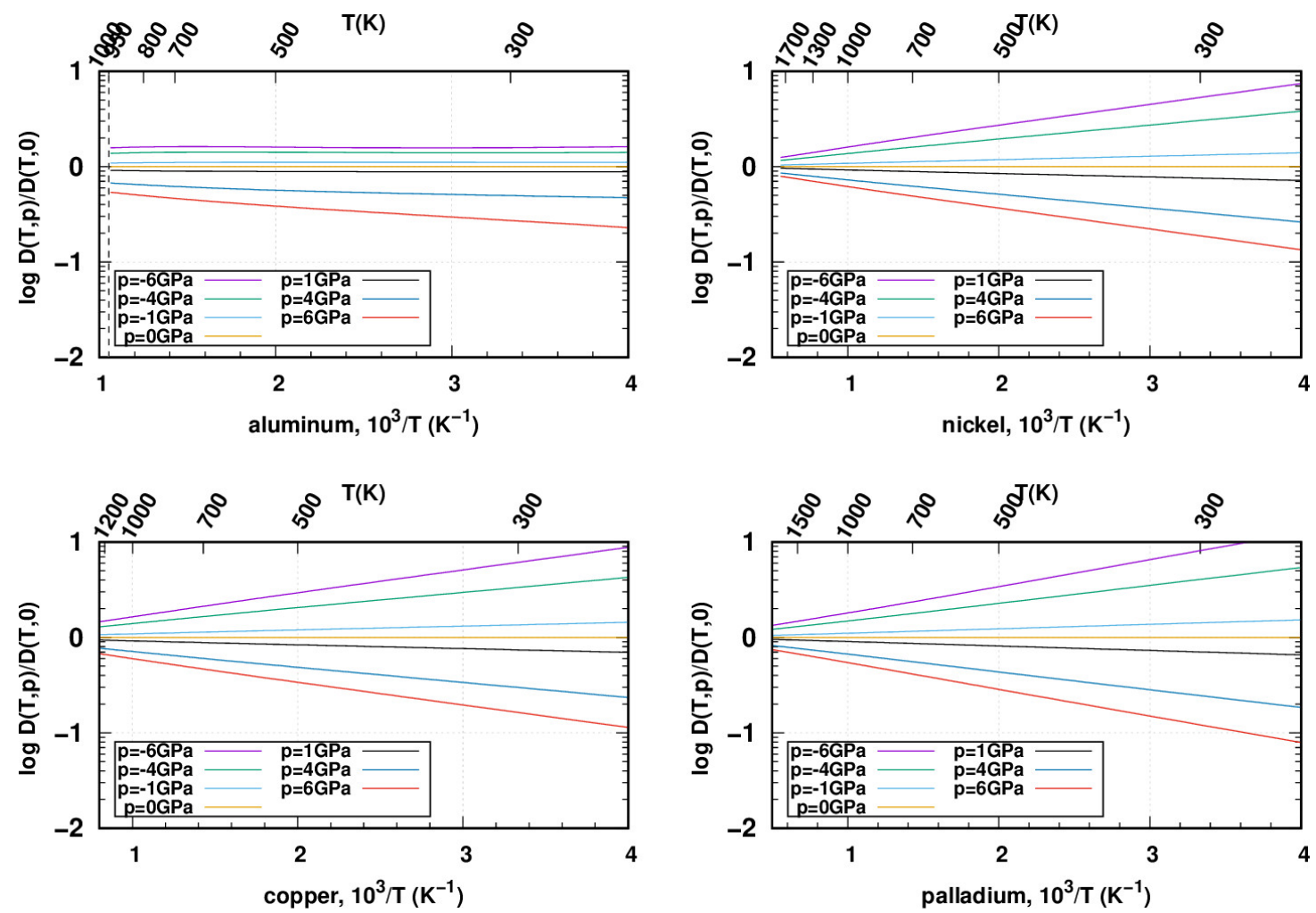

Figure 5. $D[T, p] / D[T, 0]$ (log-scale) as a function of temperature under different pressures in $\mathrm{Al}, \mathrm{Cu}, \mathrm{Ni}$ and $\mathrm{Pd}$ systems. 
significant effect on hydrogen concentration: an insertion enthalpy variation of $0.1 \mathrm{eV}$ requires a strong loading, i.e. above $\pm 4 \mathrm{GPa}$. A qualitative comparison shows that, contrary to what can be found in the Al system, where the ZPE reduces the energy difference between interstitial configurations thus modifying the relative site stability with pressure, the other systems do not exhibit this stability switch.

The evolution of hydrogen concentration and diffusivity as a function of temperature can now be analyzed. To show the effect of pressure, the ratios $C_{H}[T, p] / C_{H}[T, 0]$ and $D[T, p] / D[T, 0]$ were plotted in Figs. 4 and 5 , for different values of $p$ in the four systems, using the slopes calculated with the elastic theory listed in Tables IV and V, and Eq. 1 and Eq. 2: as hydrogen concentration and diffusivity respectively. As expected, hydrogen solubility and diffusivity increase (resp. decrease) when the system is exposed to negative (resp. positive) pressures. This is related to the fact that the activation volumes for diffusion are both positive, $\Delta V^{o-t}=2.01 \AA^{3}$ and $\Delta V^{t-o}=0.23 \AA^{3}$. It can be seen that, for all fcc systems, stress induces a similar effect on hydrogen diffusivity, except when the $\mathrm{Al}$ system is under negative pressures, as depicted in Fig. 5. The ratio $D[T, p] / D[T, 0]$ is almost constant. This is due to the fact that the energy barrier to go from $o$ to $t$ is low and decreases rapidly to zero as pressure rises.

It can be seen that for pressures in the range of \pm $1 \mathrm{GPa}$, the effect is weak. Under traction, it can be noticed that $\mathrm{H}$ atoms diffuse faster. For example, under a stress of $6 \mathrm{GPa}$ at room temperature, diffusion is at least one order of magnitude higher, this acceleration is however significantly reduced at higher temperatures. It can therefore be deduced that if hydrostatic loading does modify insertion energies and migration barriers, the effect occurs under high stresses only.

\section{B. $[001]$ and $[100]+[010]$ loadings}

The cases of [001] uni-axial and $[100]+[010]$ bi-axial loadings can be treated simultaneously. Results regarding formation and migration enthalpies are plotted in Figs. 6 and 7 respectively. Again data calculated using the elasticity theory can be found in Tables IV and V (without the ZPE).

First, it can be noted that for the two loads, the complete calculation performed using DFT is in excellent agreement with the model. The effect of $\sigma$ is weak however. This is due to the low values of the $\mathcal{P}$ components. Then, as expected from Eqs. 22, the lattice parameters also evolve with loading, Fig. 6 and 7 (bottom). The theory of elasticity thus reproduces this evolution accurately. A contraction can be noted perpendicular to the load, which will induce a symmetry break in the diffusion coefficient of $\mathrm{H}$.

The stress dependence of insertion and, to a lesser extent, of migration enthalpies is similar among all four
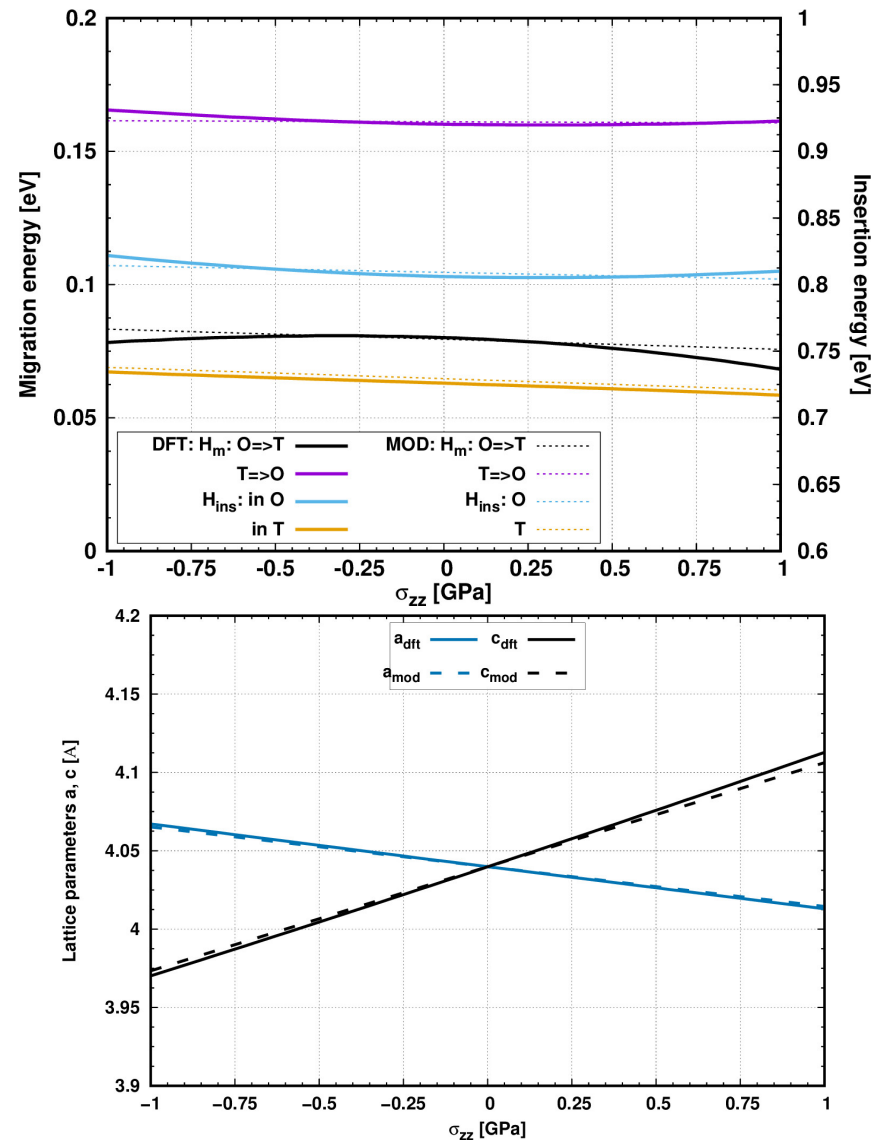

Figure 6. Evolution of formation and migration enthalpies (top) and lattice parameters (bottom) as a function of stress, $\sigma_{z z}$, in the case of uni-axial [001] stress.

metals. Concerning the symmetry break induced by stress, it results from the symmetry breaking of lattice parameters as explained above. For example, at $1 \mathrm{GPa}$, the anisotropy, calculated using Eq. 10, is equal to about $3 \%$. Anisotropy is therefore weak but present.

\section{C. [111] loading}

In this case, DFT results and the elasticity model are again in excellent agreement. Comparison between DFT simulations and the elasticity theory is depicted in Fig. 8. One notes that the elasticity captures the physics perfectly. The strain induces a small symmetry break in the dense planes. Compared to a [001] loading, a loading along [111] induces a smaller effect on anisotropy. This is because for all systems investigated, the stiffness coefficient $\mathcal{C}_{44}$ used in Eq. 13 is smaller than the shear modulus $\mathcal{C}^{\prime}$ used in Eq. 8, see Table II.

Energy barriers change very little, even for large values of $\sigma_{111}$. By taking into account the effect of the relation given by Eq. 12 and 13, and by using the data listed in Table V, the effect of stress on anisotropy can be quantified, see Fig. 9. At low temperature, anisotropy is equal 

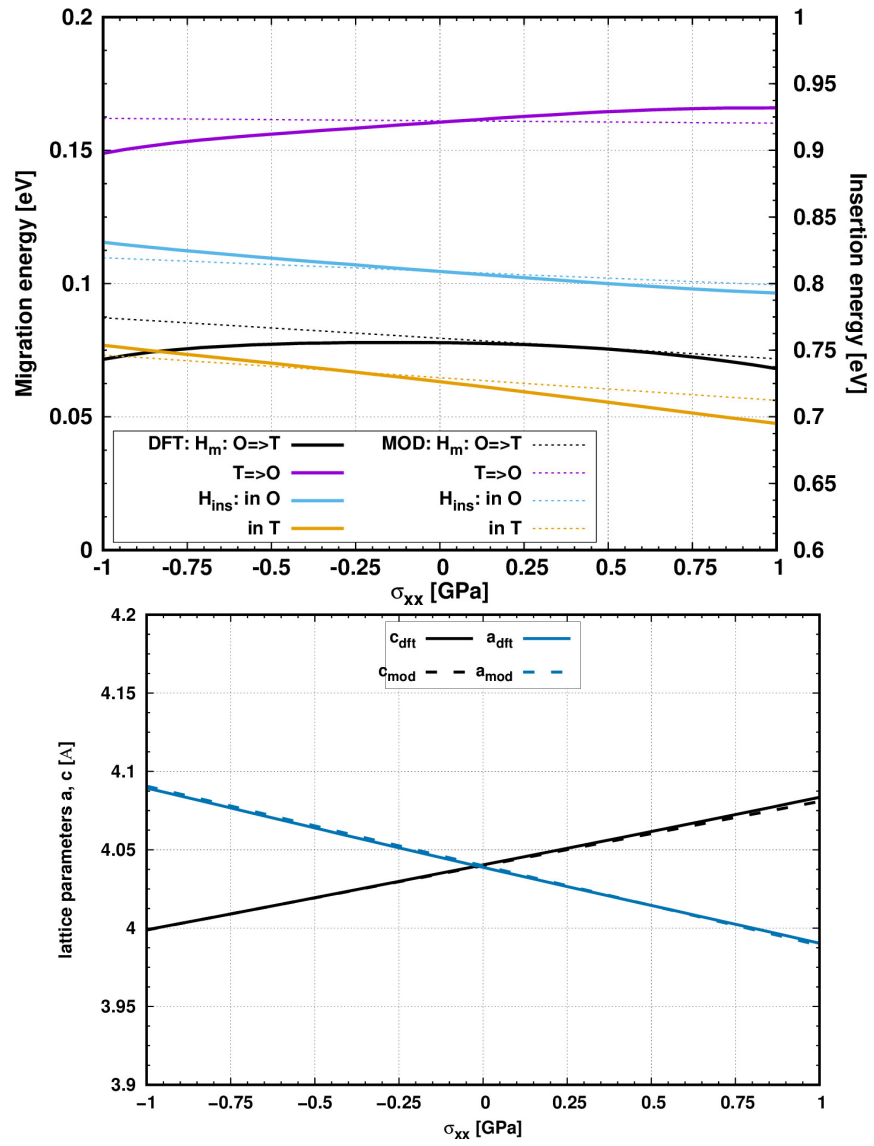

Figure 7. Evolution of formation and migration enthalpies (top) and lattice parameters (bottom) as a function of the stress component, $\sigma_{x x}$, in the case of $[100]+[010]$ stress.

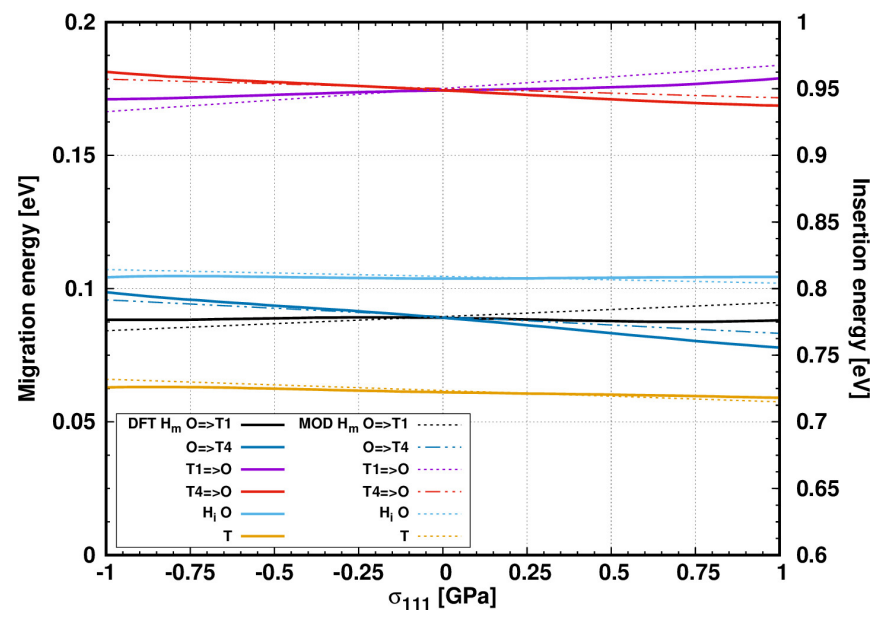

Figure 8. Evolution of formation and migration enthalpies as function of loading $\sigma_{111}$.

to 2 (the most favorable case to observe anisotropy), which means that diffusion is, at most, twice as fast in the traction direction [111].

\section{Tri-axial loading}

This case was studied solely based on the elasticity theory; no DFT calculations were performed. In case of a triaxial loading, the three diagonal components of the stress tensor differ $-\sigma_{x x} \neq \sigma_{y y} \neq \sigma_{z z}$ and the off-diagonal components are null. As the diagonal components differ, the four jumps (along [111], [111], [1111] and [11̄i] directions) should have different migration enthalpies under tri-axial stress. Nevertheless, the corresponding migration volume tensors all share the same diagonal component, i.e. $\Delta \mathcal{V}_{11}$. From Eq. 19, the effect of a tri-axial stress on the migration enthalpy does not depend on the type of jump. This is true for $o-t$ and for $t$-o jumps. The variation in migration enthalpy writes as:

$$
\Delta H_{\mathrm{m}}=-\Delta \mathcal{V}_{11}\left(\sigma_{x x}+\sigma_{y y}+\sigma_{z z}\right) .
$$

We can see that the effect of the normal stresses is additive. It is thus a function of the hydrostatic pressure only $p=-\frac{1}{3}\left(\sigma_{x x}+\sigma_{y y}+\sigma_{z z}\right)$. Results are given in Appendix A.

\section{E. Shear loading}

Lastly, the effect of pure shear loading is discussed based solely on the elasticity theory. Data are summarized in Tables IV and V. Shear loading does not induce any change in the insertion enthalpy, $\Delta H_{\text {ins }}=0$, because the strain of $\mathrm{H}$ insertion is purely due to dilatation. The slope of the migration enthalpy however shows an opposite change depending on the direction of the jump. In the $[111]$ and $[11 \overline{1}]$ directions migration enthalpy increases with stress magnitude, whereas it decreases in the [1111] and [1ํ11] directions.

From the diffusion equation for small distortion, the effect of a distortion on diffusion is here again, relatively weak.

\section{CONCLUSION}

This manuscript presents a comprehensive study of the solubility and diffusivity of hydrogen in fcc systems using the theory of point defect elasticity. First-principles calculations were used to test the validity of the theory of elasticity for which only a few parameters are required. DFT simulations were also used to provide accurate data used in the elasticity theory calculations. Different types of stresses were considered in this study: hydrostatic loading, uni-axial loading ([001] and [111]), bia-xial and tri-axial loadings, and shear stress. In each case, the exact interstitial diffusion equations were proposed and diffusion anisotropy was investigated. Results showed that, in many cases, diffusion anisotropy is only controlled by the symmetry break in lattice parameters. This is specific to fcc systems. 

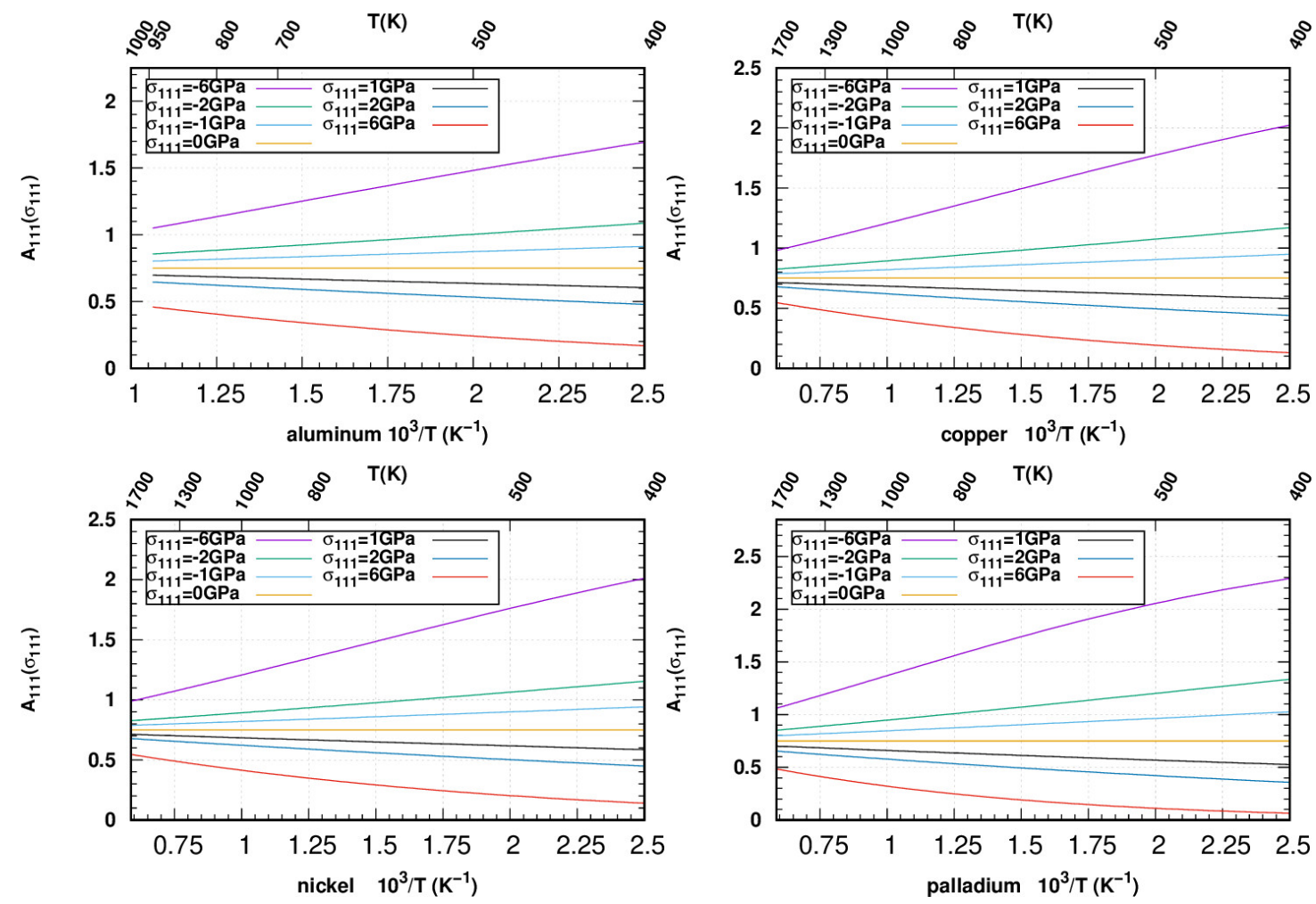

$$
\therefore
$$

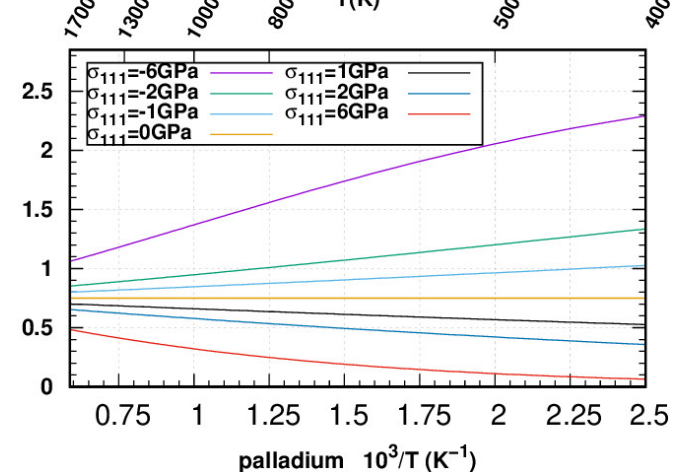

Figure 9. Evolution of anisotropy of hydrogen diffusivity as a function of loading $\sigma_{111}$.

In the different cases studied, there is an excellent agreement between DFT simulations and the elasticity theory, and the validity range covers a wide range of stress amplitudes, until \pm 2 GPa. In the case of higher stresses, however, the elasticity theory can still be used with confidence. Results also show that the effect of stress on vibrational properties is low and can be neglected to simplify. The confrontation between the two approaches shows that, for hydrogen, the elasticity theory can be used as an excellent first-order approximation theory. More specifically, quantitatively, the effect of a mechanical stress on the solubility as well as on the diffusivity of hydrogen is relatively weak, whichever the system studied. This result is directly correlated to the low values of the components of the elastic hydrogen tensor in the different metal systems studied here. Moreover, in the case of fcc, the symmetry break in the diffusion coefficient is generally due to the breaking of the lattice parameters during deformation, which is explained by the symmetry of the elastic tensor, contrary to what can be found in bcc systems [33, 35].

The study proposed here is intended to be used and directly transposed to other interstitials $(\mathrm{C}, \mathrm{O}$ and $\mathrm{N}$, for instance) provided the atomic scale processes are identical to those presented here; otherwise adjustments will have to be made, especially to calculate diffusion coefficients.

\section{ACKNOWLEDGMENTS}

This work was performed using HPC resources from CALMIP (Grant 2019 and 2020-p0749) and GENCICINES (Grant A0040910368).

\section{Appendix A: Data}

In this Appendix, data obtained from the elasticity theory are gathered in Table IV and V.
[1] B. D. Adams, A. Chen, The role of palladium in a hydrogen economy, Materials Today 14 (6) (2011) 282289. doi:https://doi.org/10.1016/S1369-7021(11) 70143-2.

URL https://www.sciencedirect.com/science/ article/pii/S1369702111701432
[2] A. Díaz, J. Alegre, I. Cuesta, A review on diffusion modelling in hydrogen related failures of metals, Engineering Failure Analysis 66 (2016) 577-595. doi:https: //doi.org/10.1016/j.engfailanal.2016.05.019.

URL https://www.sciencedirect.com/science/ article/pii/S1350630716303193 
Table IV. Relaxation volume tensors $\left(\mathcal{V}^{\mathrm{o}, \mathrm{t}}\right.$, in $\left.\AA^{3}\right)$ and changes in insertion enthalpy $\left(\Delta H^{\mathrm{o}, \mathrm{t}}\right.$, in eV) due to stress $(p$ or $\sigma$, in GPa) for $\mathrm{H}$ in $o$ and $t$ sites. From DFT calculations with $3 \times 3 \times 3$ super-cell size (Table III) and elasticity theory of point defects.

\begin{tabular}{|c|c|c|c|c|c|}
\hline \multicolumn{6}{|c|}{$\mathrm{H}$ in octahedral sites } \\
\hline \multirow[t]{2}{*}{ stress state } & & $\mathrm{Al}$ & $\mathrm{Ni}$ & $\mathrm{Cu}$ & $\mathrm{Pd}$ \\
\hline & $\mathcal{V}^{\circ}$ & $0.87 \cdot \mathcal{I}$ & $0.74 \cdot \mathcal{I}$ & $0.89 \cdot \mathcal{I}$ & $0.87 \cdot \mathcal{I}$ \\
\hline hydrostatic & $\Delta H_{\mathrm{ins}}^{\mathrm{o}}$ & $+0.0163 p$ & $+0.0139 p$ & $+0.0167 p$ & $+0.0162 p$ \\
\hline uni-axial [001] & $\Delta H_{\mathrm{ins}}^{\mathrm{o}}$ & $-0.0054 \sigma_{z z}$ & $-0.0046 \sigma_{z z}$ & $-0.0056 \sigma_{z z}$ & $-0.0054 \sigma_{z z}$ \\
\hline uni-axial [111] & $\Delta H_{\mathrm{ins}}^{\mathrm{o}}$ & $-0.0054 \sigma_{111}$ & $-0.0046 \sigma_{111}$ & $-0.0056 \sigma_{111}$ & $-0.0054 \sigma_{111}$ \\
\hline bi-axial $[100]+[100]$ & $\Delta H_{\mathrm{ins}}^{\mathrm{o}}$ & $-0.0109 \sigma_{x x}$ & $-0.0093 \sigma_{x x}$ & $-0.0111 \sigma_{x x}$ & $-0.0108 \sigma_{x x}$ \\
\hline tri-axial & $\Delta H_{\mathrm{ins}}^{\mathrm{o}}$ & $-0.0054 \sum \sigma_{i i}$ & $-0.0046 \sum \sigma_{i i}$ & $-0.0056 \sum \sigma_{i i}$ & $-0.0054 \sum \sigma_{i i}$ \\
\hline shear (001) & $\Delta H_{\mathrm{ins}}^{\mathrm{o}}$ & 0. & 0. & 0. & 0. \\
\hline \multicolumn{6}{|c|}{$\mathrm{H}$ in tetrahedral sites } \\
\hline \multirow[t]{2}{*}{ stress state } & & $\mathrm{Al}$ & $\mathrm{Ni}$ & $\mathrm{Cu}$ & $\mathrm{Pd}$ \\
\hline & $\mathcal{V}^{\mathrm{t}}$ & $1.47 \cdot \mathcal{I}$ & $1.11 \cdot \mathcal{I}$ & $1.28 \cdot \mathcal{I}$ & $1.31 \cdot \mathcal{I}$ \\
\hline hydrostatic & $\Delta H_{\mathrm{ins}}^{\mathrm{t}}$ & $+0.0274 p$ & $+0.0208 p$ & $+0.0240 p$ & $+0.0246 p$ \\
\hline uni-axial [001] & $\Delta H_{\mathrm{ins}}^{\mathrm{t}}$ & $-0.0091 \sigma_{z z}$ & $-0.0069 \sigma_{z z}$ & $-0.0080 \sigma_{z z}$ & $-0.0082 \sigma_{z z}$ \\
\hline uni-axial [111] & $\Delta H_{\mathrm{ins}}^{\mathrm{t}}$ & $-0.0091 \sigma_{111}$ & $-0.0069 \sigma_{111}$ & $-0.0080 \sigma_{111}$ & $+0.0082 \sigma_{111}$ \\
\hline bi-axial $[100]+[100]$ & $\Delta H_{\mathrm{ins}}^{\mathrm{t}}$ & $-0.0183 \sigma_{x x}$ & $-0.0139 \sigma_{x x}$ & $-0.0160 \sigma_{x x}$ & $-0.0164 \sigma_{x x}$ \\
\hline tri-axial & $\Delta H_{\mathrm{ins}}^{\mathrm{t}}$ & $-0.0091 \sum \sigma_{i i}$ & $-0.0069 \sum \sigma_{i i}$ & $-0.0080 \sum \sigma_{i i}$ & $-0.0082 \sum \sigma_{i i}$ \\
\hline shear (001) & $\Delta H_{\mathrm{ins}}^{\mathrm{t}}$ & 0. & 0. & 0. & 0. \\
\hline
\end{tabular}

[3] C. Beachem, A new model for hydrogen-assisted cracking (hydrogen "embrittlement"), Metallurgical and Materials Transactions B 3 (2) (1972) 441-455.

[4] H. K. Birnbaum, P. Sofronis, Hydrogen-enhanced localized plasticity - a mechanism for hydrogen-related fracture, Materials Science and Engineering: A 176 (1-2) (1994) 191-202.

[5] P. Sofronis, Y. Liang, N. Aravas, Hydrogen induced shear localization of the plastic flow in metals and alloys, European Journal of Mechanics-A/Solids 20 (6) (2001) 857872.

[6] A. R. Troiano, The role of hydrogen and other interstitials in the mechanical behavior of metals, trans. ASM 52 (1960) 54-80.

[7] S. Lynch, Hydrogen embrittlement and liquid-metal embrittlement in nickel single crystals, Scripta Metallurgica 13 (11) (1979) 1051-1056.

[8] S. P. Lynch, Mechanisms and kinetics of environmentally assisted cracking: current status, issues, and suggestions for further work, Metallurgical and Materials Transactions A 44 (3) (2013) 1209-1229.

[9] R. McLellan, Z. Xu, Hydrogen-induced vacancies in the iron lattice, Scripta materialia 36 (10) (1997).

[10] Y. Fukai, The metal-hydrogen system: basic bulk properties, Vol. 21, Springer Science \& Business Media, 2006.

[11] I. Robertson, The effect of hydrogen on dislocation dynamics, Engineering Fracture Mechanics 68 (6) (2001) 671 - 692. doi:https: //doi.org/10.1016/S0013-7944(01)00011-X.

URL http://www.sciencedirect.com/science/ article/pii/S001379440100011X

[12] X. Shen, D. Connétable, D. Tanguy, Modeling segregation of hydrogen to the $\sigma_{9}-(221)-[110]$ symmetric tilt grain boundary in al, Phil. Mag. 94 (2014) 2247-2261.
[13] R. Nazarov, T. Hickel, J. Neugebauer, Ab initio study of h-vacancy interactions in fcc metals: Implications for the formation of superabundant vacancies, Phys. Rev. B 89 (2014) 144108. doi:10.1103/PhysRevB.89.144108.

URL http://link.aps.org/doi/10.1103/PhysRevB . 89. 144108

[14] D. Tanguy, Y. Wang, D. Connétable, Stability of vacancy-hydrogen clusters in nickel from first principles calculations, Acta Materialia 78 (2014) 135-143.

[15] Y. Wang, D. Connétable, D. Tanguy, Influence of trap connectivity on $\mathrm{h}$ diffusion: Vacancy trapping, Acta Materialia 103 (2016) 334-340.

[16] D. Connétable, M. David, Diffusion of interstitial species (h and o atoms) in fcc systems (al, cu, co, ni and pd): Contribution of first and second order transition states, Journal of Alloys and Compounds 772 (2019) 280 - 287. doi:https: //doi.org/10.1016/j.jallcom.2018.09.042.

URL http://www.sciencedirect.com/science/ article/pii/S0925838818332717

[17] M. David, D. Connétable, Diffusion of interstitials in metallic systems, illustration of a complex study case: aluminum, Journal of Physics: Condensed Matter 29 (45) (2017) 455703.

URL http://stacks.iop.org/0953-8984/29/i=45/a= 455703

[18] H. Momida, Y. Asari, Y. Nakamura, Y. Tateyama, T. Ohno, Hydrogen-enhanced vacancy embrittlement of grain boundaries in iron, Phys. Rev. B 88 (2013) 144107. doi:10.1103/PhysRevB.88.144107.

URL https://link.aps.org/doi/10.1103/PhysRevB. 88.144107

[19] X. Zhou, D. Marchand, D. L. McDowell, T. Zhu, J. Song, Chemomechanical origin of hydrogen trapping at grain boundaries in fcc metals, Phys. Rev. Lett. 116 (2016) 
Table V. Octahedral to tetrahedral atom jumps. Migration dipole tensor $(\Delta \mathcal{P}$, in eV) and migration volume tensor $(\Delta \mathcal{V}$, in $\left.\AA^{3}\right)$ for a jump in direction [111]. Changes in migration enthalpy $\left(\Delta H_{\mathrm{m}}\right.$, in eV) due to stress $(p$ or $\sigma$, in GPa) for various jump directions. From DFT calculations with $3 \times 3 \times 3$ super-cell size and elasticity theory of point defects.

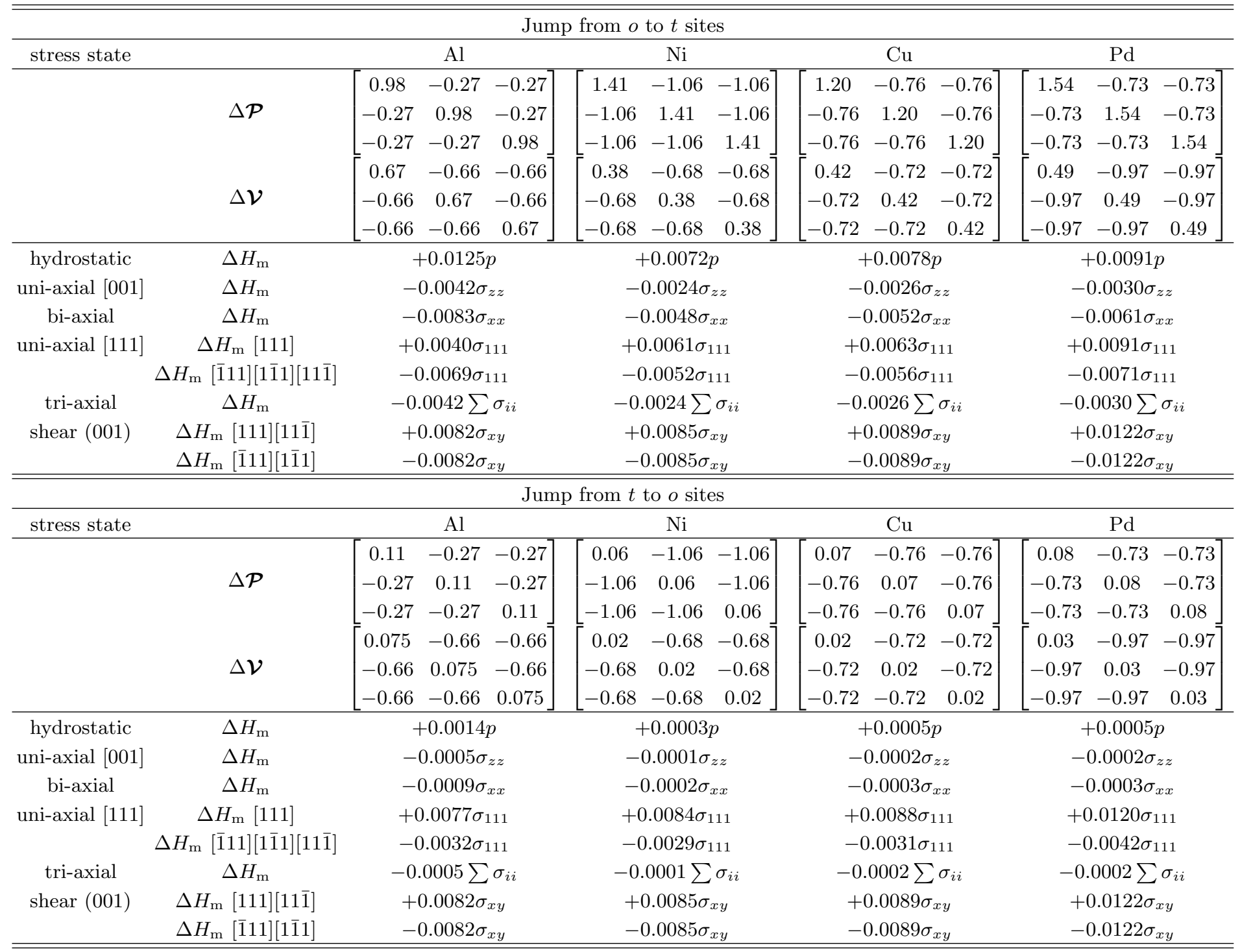

075502. doi:10.1103/PhysRevLett.116.075502.

URL https://link.aps.org/doi/10.1103/ PhysRevLett.116.075502

[20] L. Wan, W. T. Geng, A. Ishii, J.-P. Du, Q. Mei, N. Ishikawa, H. Kimizuka, S. Ogata, Hydrogen embrittlement controlled by reaction of dislocation with grain boundary in alpha-iron, International Journal of Plasticity 112 (2019) 206 - 219. doi:https: //doi.org/10.1016/j.ijplas.2018.08.013.

URL http://www.sciencedirect.com/science/ article/pii/S0749641918303103

[21] J. L. Corrigall, C. St Louis, C. E. Coleman, G. A. McRae, A chemical potential probe to determine the solubility of hydrogen in metals: An example with copper, Journal of Phase Equilibria and Diffusion (Jan 2020). doi:10. 1007/s11669-019-00776-2.

URL https://doi.org/10.1007/s11669-019-00776-2
[22] B. Amin-Ahmadi, D. Connétable, M. Fivel, D. Tanguy, R. Delmelle, S. Turner, L. Malet, S. Godet, T. Pardoen, J. Proost, D. Schryvers, H. Idrissi, Dislocation/hydrogen interaction mechanisms in hydrided nanocrystalline palladium films, Acta Materialia 111 (2016) 253 - 261. doi: https://doi.org/10.1016/j.actamat.2016.03.054. URL http://www.sciencedirect.com/science/ article/pii/S1359645416302166

[23] C. Wolverton, V. Ozolins, M. Asta, Hydrogen in aluminum: First-principles calculations of structure and thermodynamics, Phys. Rev. B 69 (2004) 144109. doi: 10.1103/PhysRevB.69.144109.

URL https://link.aps.org/doi/10.1103/PhysRevB. 69.144109

[24] D. Di Stefano, M. Mrovec, C. Elsässer, First-principles investigation of quantum mechanical effects on the diffusion of hydrogen in iron and nickel, Phys. Rev. B 92 (2015) 224301. doi:10.1103/PhysRevB.92.224301. 
URL https://link.aps.org/doi/10.1103/PhysRevB. 92. 224301

[25] H. Kimizuka, S. Ogata, M. Shiga, Mechanism of fast lattice diffusion of hydrogen in palladium: Interplay of quantum fluctuations and lattice strain, Phys. Rev. B 97 (2018) 014102. doi:10.1103/PhysRevB.97.014102.

URL https://link.aps.org/doi/10.1103/PhysRevB. 97.014102

[26] D. R. Trinkle, Diffusivity and derivatives for interstitial solutes: activation energy, volume, and elastodiffusion tensors, Philosophical Magazine 96 (26) (2016) 2714-2735. arXiv:https: //doi.org/10.1080/14786435.2016.1212175, doi:10.1080/14786435.2016.1212175.

URL https://doi.org/10.1080/14786435.2016. 1212175

[27] H.-B. Zhou, S. Jin, Y. Zhang, G.-H. Lu, F. Liu, Anisotropic strain enhanced hydrogen solubility in bcc metals: The independence on the sign of strain, Phys. Rev. Lett. 109 (2012) 135502. doi:10.1103/PhysRevLett.109.135502.

URL https://link.aps.org/doi/10.1103/ PhysRevLett.109.135502

[28] M. Iyer, V. Gavini, T. M. Pollock, Energetics and nucleation of point defects in aluminum under extreme tensile hydrostatic stresses, Physical Review B 84 (2014) 014108.

[29] D. S. Tchitchekova, J. Morthomas, F. Ribeiro, R. Ducher, M. Perez, A novel method for calculating the energy barriers for carbon diffusion in ferrite under heterogeneous stress, The Journal of Chemical Physics 141 (3) (2014) 034118. arXiv:https://doi.org/10. 1063/1.4889854, doi:10.1063/1.4889854.

URL https://doi.org/10.1063/1.4889854

[30] M. Souissi, Y. Chen, M. H. Sluiter, H. Numakura, Ab initio characterization of b, c, n, and o in bcc iron: Solution and migration energies and elastic strain fields, Computational Materials Science 124 (2016) 249 - 258. doi: https://doi.org/10.1016/j.commatsci.2016.07.037. URL http://www.sciencedirect.com/science/ article/pii/S0927025616303640

[31] P. Maugis, F. Danoix, H. Zapolsky, S. Cazottes, M. Gouné, Temperature hysteresis of the order-disorder transition in carbon-supersaturated $\alpha$-fe, Phys. Rev. B 96 (2017) 214104. doi:10.1103/PhysRevB.96.214104. URL https://link.aps.org/doi/10.1103/PhysRevB. 96. 214104

[32] P. Maugis, S. Chentouf, D. Connétable, Stresscontrolled carbon diffusion channeling in bct-iron: A mean-field theory, Journal of Alloys and Compounds 769 (2018) 1121 - 1131. doi:https: //doi.org/10.1016/j.jallcom.2018.08.060.

URL http://www.sciencedirect.com/science/ article/pii/S0925838818329335

[33] P. Maugis, D. Kandaskalov, Revisiting the pressure effect on carbon migration in iron, Materials Letters 270 (2020) 127725. doi:https: //doi.org/10.1016/j.matlet.2020.127725.

URL http://www.sciencedirect.com/science/ article/pii/S0167577X20304304

[34] P. Maugis, Giant snoek peak in ferrite due to carboncarbon strain interactions, Materialia 12 (2020) 100805. doi:https://doi.org/10.1016/j.mtla.2020.100805. URL http://www.sciencedirect.com/science/ article/pii/S2589152920302210
[35] E. Clouet, S. Garruchet, H. Nguyen, M. Perez, C. S. Becquart, Dislocation interaction with c in alpha-fe: A comparison between atomic simulations and elasticity theory, Acta Materialia 56 (14) (2008) 3450 - 3460. doi: https://doi.org/10.1016/j.actamat.2008.03.024.

URL http://www.sciencedirect.com/science/ article/pii/S1359645408002218

[36] C. Varvenne, F. Bruneval, M.-C. Marinica, E. Clouet, Point defect modeling in materials: Coupling ab initio and elasticity approaches, Phys. Rev. B 88 (2013) 134102. doi:10.1103/PhysRevB.88.134102.

URL https://link.aps.org/doi/10.1103/PhysRevB. 88.134102

[37] C. Varvenne, E. Clouet, Elastic dipoles of point defects from atomistic simulations, Phys. Rev. B 96 (2017) 224103. doi:10.1103/PhysRevB.96.224103.

URL https://link.aps.org/doi/10.1103/PhysRevB. 96.224103

[38] P.-W. Ma, S. L. Dudarev, Effect of stress on vacancy formation and migration in body-centeredcubic metals, Phys. Rev. Materials 3 (2019) 063601. doi:10.1103/PhysRevMaterials.3.063601.

URL https://link.aps.org/doi/10.1103/ PhysRevMaterials.3.063601

[39] D. Connétable, P. Maugis, Effect of stress on vacancy formation and diffusion in fcc systems: Comparison between dft calculations and elasticity theory, Acta Materialia 200 (2020) 869 - 882. doi:https: //doi.org/10.1016/j.actamat.2020.09.053.

URL http://www.sciencedirect.com/science/ article/pii/S1359645420307473

[40] M. I. Aroyo, J. M. Perez-Mato, C. Capillas, Bilbao crystallographic server: I. databases and crystallographic computing programs, Zeitschrift für Kristallographie Crystalline Materials 221 (2009) 15-27. doi:https: //doi.org/10.1524/zkri.2006.221.1.15.

[41] A. Sieverts, The absorption of gases by metals, Zeitschrift für Metallkunde 21 (1929) 37-46.

[42] E. Wimmer, W. Wolf, J. Sticht, P. Saxe, C. B. Geller, R. Najafabadi, G. A. Young, Temperature-dependent diffusion coefficients from ab initio computations: Hydrogen, deuterium, and tritium in nickel, Phys. Rev. B 77 (2008) 134305. doi:10.1103/PhysRevB.77.134305.

URL https://link.aps.org/doi/10.1103/PhysRevB. 77.134305

[43] U. Landman, M. F. Shlesinger, Stochastic theory of multistate diffusion in perfect and defective systems. i. mathematical formalism, Phys. Rev. B 19 (1979) 6207-6219. doi:10.1103/PhysRevB.19.6207.

URL http://link.aps.org/doi/10.1103/PhysRevB. 19.6207

[44] H. Eyring, The activated complex in chemical reactions, The Journal of Chemical Physics 3 (2) (1935) 107-115. arXiv:https://doi.org/10.1063/1.1749604, doi:10.1063/1.1749604.

URL https://doi.org/10.1063/1.1749604

[45] P. H. Dederichs, K. Schroeder, Anisotropic diffusion in stress fields, Phys. Rev. B 17 (6) (1978) 2524-2536. doi:10.1103/PhysRevB.17.2524.

URL https://link.aps.org/doi/10.1103/PhysRevB. 17.2524

[46] R. W. Balluffi, Introduction to elasticity theory for crystal defects, Cambridge University Press, 2012. doi: 10.1017/CB09780511998379. 
[47] D. Bacon, D. Barnett, R. Scattergood, Anisotropic continuum theory of lattice defects, Prog. Mater. Sci. 23 (1980) 51. doi:10.1016/0079-6425(80)90007-9. URL http://www.sciencedirect.com/science/ article/pii/0079642580900079

[48] B. Lawrence, C. W. Sinclair, M. Perez, Carbon diffusion in supersaturated ferrite : A comparison of mean-field and atomistic predictions, Model. Simul. Mater. Sci. Eng. 22 (2014) 1-17. doi:10.1088/0965-0393/22/6/065003.

[49] R. Agarwal, D. R. Trinkle, Light-element diffusion in Mg using first-principles calculations: Anisotropy and elastodiffusion, Phys. Rev. B 94 (5) (2016) 054106. doi: 10.1103/PhysRevB.94.054106.

URL https://link.aps.org/doi/10.1103/PhysRevB. 94.054106

[50] E. Clouet, C. Varvenne, T. Jourdan, Elastic modeling of point-defects and their interaction, Computational Materials Science 147 (2018) 49-63. doi:https: //doi.org/10.1016/j.commatsci.2018.01.053.

URL https://www.sciencedirect.com/science/ article/pii/S0927025618300673

[51] J. S. Wróbel, M. R. Zemła, D. Nguyen-Manh, P. Olsson, L. Messina, C. Domain, T. Wejrzanowski, S. L. Dudarev, Elastic dipole tensors and relaxation volumes of point defects in concentrated random magnetic fe-cr alloys, Computational Materials Science 194 (2021) 110435.

[52] G. Kresse, J. Hafner, Ab initio molecular dynamics for liquid metals, Phys. Rev. B 47 (1993) 558-561. doi: 10.1103/PhysRevB.47.558.

URL https://link.aps.org/doi/10.1103/PhysRevB. 47.558

[53] G. Kresse, D. Joubert, From ultrasoft pseudopotentials to the projector augmented-wave method, Phys. Rev. B 59 (1999) 1758-1775. doi:10.1103/PhysRevB.59.1758. URL https://link.aps.org/doi/10.1103/PhysRevB. 59.1758

[54] P. E. Blöchl, Projector augmented-wave method, Phys. Rev. B 50 (1994) 17953-17979. doi:10.1103/PhysRevB. 50.17953.

URL https://link.aps.org/doi/10.1103/PhysRevB. 50.17953

[55] J. P. Perdew, K. Burke, M. Ernzerhof, Generalized gradient approximation made simple, Phys. Rev. Lett. 77 (1996) 3865-3868. doi:10.1103/PhysRevLett.77.3865. URL https://link.aps.org/doi/10.1103/ PhysRevLett.77.3865

[56] H. J. Monkhorst, J. D. Pack, Special points for brillouinzone integrations, Phys. Rev. B 13 (1976) 5188-5192. doi:10.1103/PhysRevB.13.5188.
URL https://link.aps.org/doi/10.1103/PhysRevB. 13.5188

[57] C. Helrich, Modern Thermodynamics with Statistical Mechanics, Springer Berlin Heidelberg, 2008.

[58] G. Henkelman, B. P. Uberuaga, H. Jónsson, A climbing image nudged elastic band method for finding saddle points and minimum energy paths, The Journal of Chemical Physics 113 (22) (2000) 9901-9904. arXiv:https:// doi.org/10.1063/1.1329672, doi:10.1063/1.1329672. URL ttps://doi.org/10.1063/1.1329672

[59] A. Togo, F. Oba, I. Tanaka, First-principles calculations of the ferroelastic transition between rutile-type and cacl $_{2}$-type $\mathrm{sio}_{2}$ at high pressures, Phys. Rev. B 78 (2008) 134106. doi:10.1103/PhysRevB.78.134106.

URL https://link.aps.org/doi/10.1103/PhysRevB. 78.134106

[60] C. Kittel, Introduction to Solid State Physics, Wiley, New York, 1996.

[61] J. R. Neighbours, F. W. Bratten, C. S. Smith, The elastic constants of nickel, Journal of Applied Physics 23 (4) (1952) 389-393. doi:http: //dx.doi.org/10.1063/1.1702218.

URL http://scitation.aip.org/content/aip/ journal/jap/23/4/10.1063/1.1702218

[62] H. Ledbetter, E. Naimon, Elastic properties of metals and alloys. ii, copper, J. Phys.Chem. Ref. Data 3 (1974) 897.

[63] J. Neighbours, C. S. Smith, The elastic constants of copper alloys, Acta Metallurgica 2 (4) (1954) 591 - 596. doi: http://dx.doi.org/10.1016/0001-6160 (54)90193-5. URL http://www.sciencedirect.com/science/ article/pii/0001616054901935

[64] J. A. Rayne, Elastic constants of palladium from 4.2-300k, Phys. Rev. 118 (1960) 1545-1549. doi:10.1103/PhysRev.118.1545.

URL http://link.aps.org/doi/10.1103/PhysRev . 118.1545

[65] L. Katz, M. Guinan, R. J. Borg, Diffusion of $\mathrm{h}_{2}, \mathrm{~d}_{2}$, and $\mathrm{t}_{2}$ in single-crystal ni and cu, Phys. Rev. B 4 (1971) 330341. doi:10.1103/PhysRevB.4.330.

URL https://link.aps.org/doi/10.1103/PhysRevB. 4.330

[66] H. Katsuta, R. Farraro, R. McLellan, The diffusivity of hydrogen in palladium, Acta Metallurgica 27 (7) (1979) 1111 - 1114. doi:https: //doi.org/10.1016/0001-6160(79)90128-7.

URL http://www.sciencedirect.com/science/ article/pii/0001616079901287 